\title{
Decoherence dynamics estimation for superconducting gate-model quantum computers
}

\author{
Laszlo Gyongyosi ${ }^{1,2,3}$
}

Received: 1 February 2020 / Accepted: 27 August 2020 / Published online: 1 October 2020

(c) The Author(s) 2020

\begin{abstract}
Superconducting gate-model quantum computer architectures provide an implementable model for practical quantum computations in the NISQ (noisy intermediate scale quantum) technology era. Due to hardware restrictions and decoherence, generating the physical layout of the quantum circuits of a gate-model quantum computer is a challenge. Here, we define a method for layout generation with a decoherence dynamics estimation in superconducting gate-model quantum computers. We propose an algorithm for the optimal placement of the quantum computational blocks of gate-model quantum circuits. We study the effects of capacitance interference on the distribution of the Gaussian noise in the Josephson energy.
\end{abstract}

Keywords Gate-model quantum computers · Quantum computers · Quantum computations

\section{Introduction}

Quantum computers exploit the fundamentals of quantum mechanics [1-14], making it possible to solve problems more efficiently than with any possible traditional computer [8-25]. The information processing network of a gate-model quantum computer uses traditionally uninterpretable phenomena such as quantum superposition or quantum entanglement [26-36]. The quantum computations are performed on some initial quantum states via a sequence of unitary operators [30,37-49]. The unitary operators

Laszlo Gyongyosi

gyongyosi@hit.bme.hu

1 School of Electronics and Computer Science, University of Southampton, Southampton SO17 $1 \mathrm{BJ}, \mathrm{UK}$

2 Department of Networked Systems and Services, Budapest University of Technology and Economics, Budapest 1117, Hungary

3 MTA-BME Information Systems Research Group, Hungarian Academy of Sciences, Budapest 1051, Hungary 
can formulate a larger unit called unitary block. A quantum circuit integrates these unitary blocks with a measurement array to measure the resulting quantum systems [24,50-62]. In a quantum computer architecture, an important issue is the development of entangled connections between the quantum systems of the quantum circuit $[15,17-$ 25]. A practical way to establish entangled connection is to use entangling gates in a gate-model quantum computer [38], along with some constraints on the circuit depths of the quantum circuits [15,17-25]. For near-term quantum computers, superconducting architectures $[1,63]$ and gate-model quantum computing [15,17-26,38,64,65] provide a well-implementable model. The development of experimental implementations of gate-model quantum computers has already been started [15,17-20,38], and the results look promising to arrive in the commercial quantum computers in the near future. The superconducting qubit architectures [1,63] allow a straightforward experimental implementation in the NISQ (noisy intermediate scale quantum) technology era [2], with non-error-corrected qubits to realize quantum simulations and quantum algorithms. In the physical layout of a gate-model quantum computer, the quantum states and quantum gates (unitary operators) are positioned in space such that the quantum gates are constrained by some hardware restrictions. Specifically, in the quantum circuit computation model of gate-model quantum computers, several hardware restrictions have to be satisfied simultaneously. A particular constraint is that some quantum gates have to be applied in multiple rounds [38]. Another constraint could be the preservation of some entangled connections between the selected gates, or the connection topology in the quantum circuit. In our approach, we assume that these constraints are present in the development phase; thus, the quantum circuits are evolved with respect to the hardware restrictions of gate-model quantum computers. Another important aspect is the application of gate-model quantum computations in near-term quantum devices of the quantum Internet [30-32,66-120].

Here, we define a method for quantum circuit layout generation for gate-model quantum computers. The layout generation is achieved through several algorithms, and we define a grid structure ${ }^{1}$ for the development of gate-model quantum circuits. The quantum circuits integrate a number of unitary computational blocks, and each unitary block consists of unitary quantum gates. In the physical layout, each quantum circuit has a particular depth (circuit depth) and a number of output wires. The grid structure encodes the physical layer placement of the unitary blocks, and it provides a tool to find the optimal placement of the unitary blocks. The grid structure can be used to iterate on the possible placements until the optimal placement is determined: The optimal placement minimizes the area of the quantum circuit such that the connections and the entanglement between the blocks are preserved. The optimal grid and the optimal placement are in a one-to-one correspondence relation. We define an algorithm for the determination of the optimal placement from an arbitrary input placement. First, the

\footnotetext{
1 The grid structure model is motivated by the space-time volume of a quantum circuit computation in a gate-model quantum computer setting [38]. In this particular model, an array of qubits is arranged on a grid with a particular height and depth. The qubit array is initialized by preparing the quantum state of each qubit. In a qubit gate-model setting, a layer of 1-qubit gates and a layer of 2-qubit gates are applied on the qubits. In the gate structure, the 2-qubit gates are applied in several rounds due to hardware restrictions, because as a constraint, one qubit cannot participate in two gates simultaneously. Note that in the grid structure model, as the circuit depth increases above a critical limit, the qubits become entangled with each other at some particular setting, see also [38].
} 
input placement is converted into a set of grids that encodes different placements. Then, the optimal placement is found in the form of a grid, using this set of grid instances. We prove the optimality of the method and extend it with a decoherence analysis. We define a decoherence estimation method for charge qubit [121] structures $^{2}$ (a charge qubit circuit integrates Josephson junctions and capacitor elements). In the system model, the charge qubit circuits are integrated into a multilayer circuit structure, with a different number of circuits in each layer. We study the capacitor interference in the multilayer quantum circuit structure and prove its effects on the decoherence dynamics. The decoherence of a particular charge qubit circuit is modeled by a Gaussian noise in the Josephson energy. Gaussian noise is a standard model of noise in the control parameters in a superconducting charge qubits. Particularly, an imperfect control impacts the qubit dynamics, since it induces a Gaussian noise in the Josephson energy. A detailed study on the Gaussian noise and its impacts in the Josephson energy can be found in [128].

The novel contributions of our manuscript are as follows:

1. We define a decoherence estimation method for charge qubit structures. The charge qubit circuits are integrated into a multilayer circuit structure in a superconducting quantum computer, with a different number of circuits in each layer.

2. We study the capacitor interference in the multilayer quantum circuit structure and prove its effects on the decoherence dynamics. The decoherence of a particular charge qubit circuit is modeled by a Gaussian noise in the Josephson energy.

3. We define a method for quantum circuit layout generation for gate-model quantum computers. We define a grid data structure for the development of gate-model quantum circuits. The grid structure encodes the physical layer placement of the unitary blocks.

This paper is organized as follows. Section 3 gives the problem statement. In Sect. 3, the layout generation framework is proposed. In Sect. 4, the decoherence estimation model is defined. Finally, Sect. 5 concludes the paper. Supplemental information is included in Appendix.

\section{Problem statement}

The problems to be solved are as follows.

Problem 1 Define a data structure for an optimal physical layer placement of the unitary blocks.

Problem 2 Evaluate the capacitor interference in multilayer superconducting quantum circuits.

Problem 3 Prove the effects of capacitor interference on decoherence in superconducting charge qubit circuits.

\footnotetext{
${ }^{2}$ Decoherence is typically considered through the environmental interactions in circuit QED and cavity QED settings. A charge qubit is a particular type of superconducting qubit. Compared to other solid-state implementations, the superconducting gap protects the condensate from the environment to some extent [121]. In the proposed model, the decoherence is associated with the interference of capacitors. Note that decoherence in a charge qubit setting can be connected to other sources, such as imperfect voltage pulses applied to the gates [121]. These cases are well studied in the literature, and we suggest [122-127].
} 
The resolutions to Problems 1-3 are proposed in the theorems and lemmas of the manuscript. The steps are detailed in the Algorithms.

\section{Methods}

In this section, we define a grid data structure for the optimal physical layer placement of entangled quantum circuits. The optimality requires that the quantum circuit area and the length of quantum wires are minimized such that the entangled connections defined by the developmenter are preserved. The grid structure is used to determine the optimal placement from a non-optimal input placement. The non-optimal placement is converted into a set of grids, and after some manipulations, the optimal grid is determined that encodes the optimal physical layer placement.

\subsection{Grid structure}

Proposition 1 There exists a d-dimensional, $N$ size grid $G^{d}$ to encode the $\mathcal{P}$ physical placement of the $m$ unitary computational blocks $U=\left\{U_{1}, U_{2}, \ldots, U_{m}\right\}$ of a quantum circuit $Q G$, with $k$ entangled connections between the computational blocks.

Let $U=\left\{U_{1}, U_{2}, \ldots, U_{m}\right\}$ refer to the set of $m$ unitary blocks, where the $i$ th unitary block, $U_{i}$, integrates unitary operators such that the circuit depth of $U_{i}$ is $D_{i}$, where the number of quantum wires $N_{i}$ is evaluated as

$$
N_{i}=\max \left(n_{i}^{\text {in }}, n_{i}^{\text {out }}\right), 1 \leq i \leq m
$$

where $n_{i}^{i n}$ and $n_{i}^{\text {out }}$ are the number of input and output quantum wires of $U_{i}$, and area $A_{i}$ as

$$
A_{i}=D_{i} N_{i}, 1 \leq i \leq m \text {. }
$$

To achieve a physical layer placement $\mathcal{P}$ of the unitary blocks, let $\left(x_{i}, y_{i}\right)$ refer to the bottom-left corner of $U_{i}$ [129-133]. For an optimal placement $\mathcal{P}^{*}$, the following conditions have to be satisfied: First, the total area $A$ of the quantum circuit is minimized, with no overlap between the modules, such that all unitary blocks are compacted in both $x$ and $y$ directions; thus,

$$
A=\sum_{i=1}^{m} D_{i} N_{i}=\min \kappa\left(\mathcal{P}^{*}\right),
$$

where $\kappa\left(\mathcal{P}^{*}\right)$ is the minimum bounding rectangle of $\mathcal{P}^{*}$.

Second, the length $\ell$ of quantum wires of $\mathcal{P}^{*}$ is minimized:

$$
\ell=\sum_{i=1}^{m} w_{i}=\min \beta\left(\mathcal{P}^{*}\right),
$$

where $w_{i}$ is the total quantum wire length associated with $U_{i}$ and $\beta\left(\mathcal{P}^{*}\right)$ is the sum of the half-bounding box of interconnections between the unitary blocks. 
Third, all of these conditions have to be satisfied such that the set of $k$ entangled connections

$$
E=\left\{E_{1}, E_{2}, \ldots, E_{k}\right\}
$$

between the blocks is preserved in the optimal placement $\mathcal{P}^{*}$.

The construction method of the $G^{d}, d$-dimensional, $N$ size grid for the optimal placement $\mathcal{P}^{*}$ with constraints (3), (4), and (5) is summarized in Procedure 1.

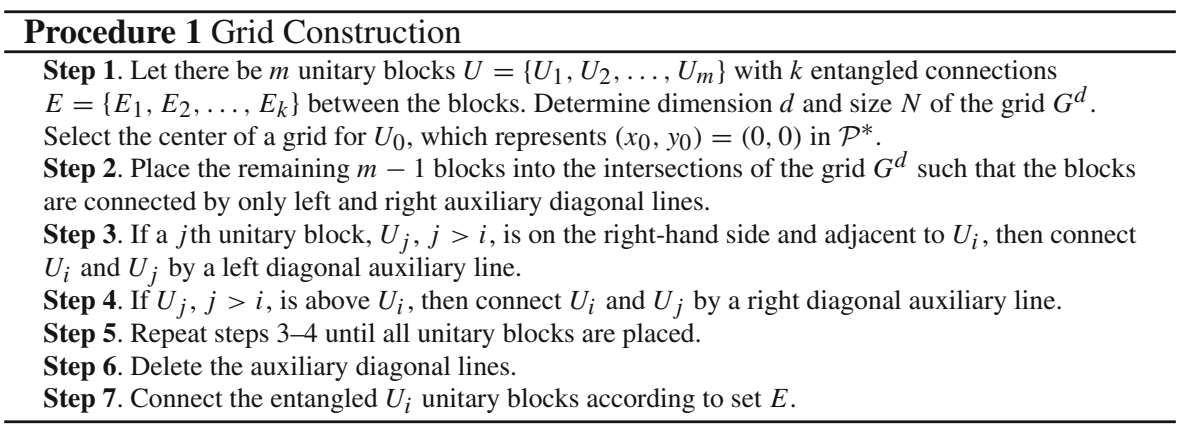

\subsubsection{Discussion}

A detailed description of Procedure 1 is as follows.

In Step 1, the development constraints are defined. The aim of the procedure is to determine an optimal physical layer placement of the unitary blocks such that the entangled connections between the particular blocks are preserved.

Step 2 assures that the first unitary block $U_{0}$ is placed at the center of a particular grid, while the other blocks can be placed only at the intersections of $G^{d}$. The unitary blocks are connected by auxiliary diagonal lines that identify the placement of the blocks in the physical layer minimization process. A given $U_{i}$ can be connected by at most two auxiliary diagonal lines: One left, $U_{i+1}$, and one right unitary block, $U_{i+2}$, are allowed [129-133].

Step 3 achieves the $\mathcal{P}^{*}$ physical layer placement of the unitary block $U_{j}$ with respect to $U_{i}$, such that $[132,133]$

$$
x_{j}=x_{i}+D_{i}
$$

in $\mathcal{P}^{*}$, where $x_{i}$ and $x_{j}$ are the $x$-coordinates of the bottom-left corner of $U_{i}$ and $U_{j}$, while $D_{i}$ is the circuit depth of $U_{i}$.

Step 4 achieves the physical layer placement of the unitary block $U_{j}$ with respect to $U_{i}$, such that

$$
x_{j}=x_{i}
$$

in $\mathcal{P}^{*}$. Note that (6) and (7) ensures that $y_{j} \in\left[x_{j}, x_{j}+D_{j}\right]$.

Step 5 achieves the placement of all unitary blocks of the quantum circuit.

Step 6 is verified by the fact that the grid structure of $G^{d}$ uniquely identifies the placement $\mathcal{P}^{*}$ (see Lemma 1). 
Step 7 ensures the entangled circuit topology between the particular blocks.

The proof is concluded here.

Additional information is included in Sect. 1.

\subsubsection{Optimality}

Lemma 1 The $G^{d}$ grid structure provides an optimal structure for the placement of the $m$ computational blocks with the preservation of the $k$ entangled connections of a QG quantum circuit.

Proof The optimality of the $G^{d}$ grid structure means that there is a one-to-one correspondence between an optimal placement $\mathcal{P}^{*}$ and the optimal grid $G^{d *}$. As a corollary, the optimal placement of the unitary blocks can be determined by a packing procedure in the grid structure.

The optimality in the physical layer is interpretable in terms of packing area. In $G^{d}, U_{0}$ identifies a unique base bottom-left coordinate $\left(x_{0}, y_{0}\right)=(0,0)$ in $\mathcal{P}^{*}$, and all remaining computational blocks are adjacent to at least one other module, if it exists.

For the determination of a $U_{j}$ left-diagonal connection of $U_{0}$, let us assume as the first case that there is a set $\mathcal{S}_{r}$ of unitary blocks placed right adjacent to $U_{0}[132,133]$. In this case, the block $U^{\prime}$ with the smallest $y$-coordinate from $\mathcal{S}_{r}$ is also unique; therefore, $U_{0}$ and $U^{\prime}$ can be connected by a left-diagonal line in the grid; thus, $U_{j}=U^{\prime}$. Then, as a second case, let us assume that $\mathcal{S}_{r}=\emptyset$. In this case, there is no left-diagonal line from $U_{0}$ and any other computational block; thus, $U_{j}=\emptyset$.

For the determination of a $U_{j}$ right-diagonal connection of $U_{0}$, let $\mathcal{S}_{a}$ be the set of unitary blocks above $U_{0}[132,133]$. Then, a right-diagonal connection $U_{j}$ from $U_{0}$ identifies the block $U^{\prime}$ with the smallest $y$-coordinate from $\mathcal{S}_{a}$ such that $x^{\prime}=x_{0}$, where $x^{\prime}$ is the $x$-coordinate of $U^{\prime}$. Assuming that $\mathcal{S}_{a} \neq \emptyset$, there always exists such a unique $U^{\prime}$, since $\mathcal{P}^{*}$ is optimal; thus, $U_{j}=U^{\prime}$. If $\mathcal{S}_{a}=\emptyset$, then such a unique $U^{\prime}$ does not exist; thus, $U_{j}=\emptyset$.

These assumptions can be extended to all computational blocks; therefore, there is a one-to-one correspondence between an optimal placement $\mathcal{P}^{*}$ and its unique grid structure $G^{d *}$ [129-133].

As a corollary, in the physical layer, the coordinates of all computational blocks can be uniquely determined from $G^{d *}$ using the construction rules (6) and (7).

Note that by some fundamental theory on tree data structures [129-133], the possible combinations for a $d=2$-dimensional grid $G^{2}$ at $m$ unitary blocks is as

$$
\mathcal{O}\left(\frac{m ! 2^{2 m}}{m^{1.5}}\right)
$$

The proof is concluded here.

\subsection{Grid set generation}

Let us assume that the developmenter inputs a placement $\mathcal{P}$. The problem then is to find a set of different $G^{d}$ grid encodings that encode the input placement. Then, the 
optimal grid $G^{d *}$ has to be determined for the optimal placement $\mathcal{P}^{*}$. For the resolution of the former problem, we define algorithm $\mathcal{A}_{1}$, while for the latter problem, we define algorithm $\mathcal{A}_{2}$.

Lemma 2 For an input placement $\mathcal{P}$, there exists a set $\mathcal{S}_{\mathcal{P}}\left(G^{d}\right):\left\{G_{1}^{d}, \ldots, G_{c}^{d}\right\}$ of $c$ different grid instances that encode $\mathcal{P}$ such that the $k$ entangled connections are satisfied.

Proof In an input placement $\mathcal{P}$, a $U_{i}$ unitary block is represented by a box with particular $x_{i}$ and $y_{i}$ coordinates in the bottom-left corner, along with the depth $D_{i}$ and $N_{i}$.

The aim of algorithm $\mathcal{A}_{1}$ is to convert the non-optimal input placement $\mathcal{P}$ with the particular constraints into a set $\mathcal{S}_{\mathcal{P}}\left(G^{d}\right):\left\{G_{1}^{d}, \ldots, G_{c}^{d}\right\}$ of $c$ different grids that encode $\mathcal{P}$. During the grid construction, the fact that a left-diagonal connection $U_{j}$ of a block $U_{i}$ in $G^{d}$ represents that $U_{j}$ is above $U_{i}$ in $\mathcal{P}$ can be exploited.

The steps of $\mathcal{A}_{1}$ are summarized in Algorithm 1.

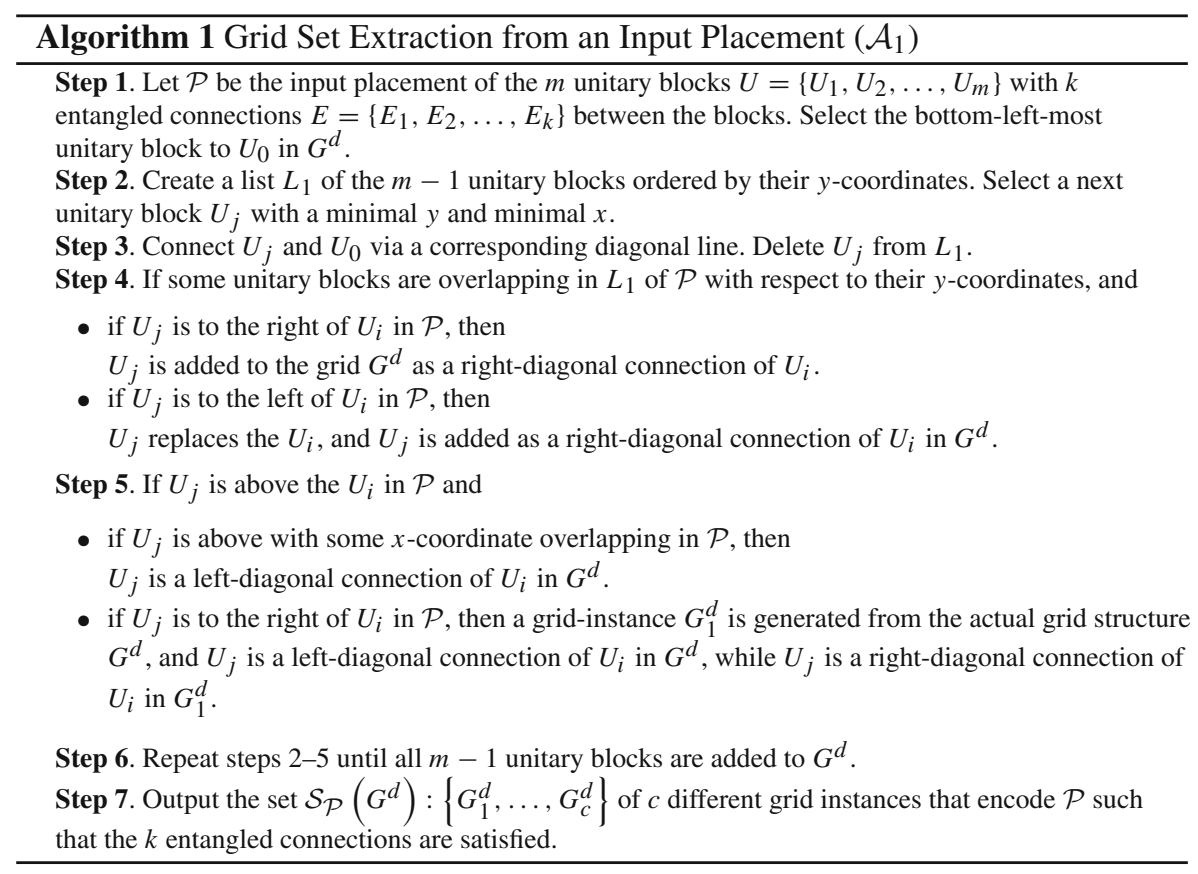

The proof is concluded here.

\subsubsection{Computational complexity}

By some fundamental assumptions, at $m$ unitary blocks, the computational complexity of $\mathcal{A}_{1}$ is

$$
\mathcal{O}\left(\mathcal{A}_{1}\right)=\mathcal{O}(m)
$$




\subsection{Optimal solution}

Theorem 1 The $G^{d *}$ optimal grid can be determined from a set $\mathcal{S}_{\mathcal{P}}\left(G^{d}\right)$ of grid instances output by algorithm $\mathcal{A}_{1}$ such that all $k$ entangled connections are preserved in $\mathcal{P}^{*}$.

Proof The aim of this step is to produce the optimal placement via the set of grid instances. Motivated by the fundamentals of simulated annealing [134,135], we define algorithm $\mathcal{A}_{2}$ to determine the optimal $G^{d *}$ grid from the set $\mathcal{S}_{\mathcal{P}}\left(G^{d}\right)$ of grid instances output by $\mathcal{A}_{1}$ such that all input constraints (gate connections, entangled connections, or decoherence estimation; see Sect. 4) are satisfied.

The steps of $\mathcal{A}_{2}$ are summarized in Algorithm 2.

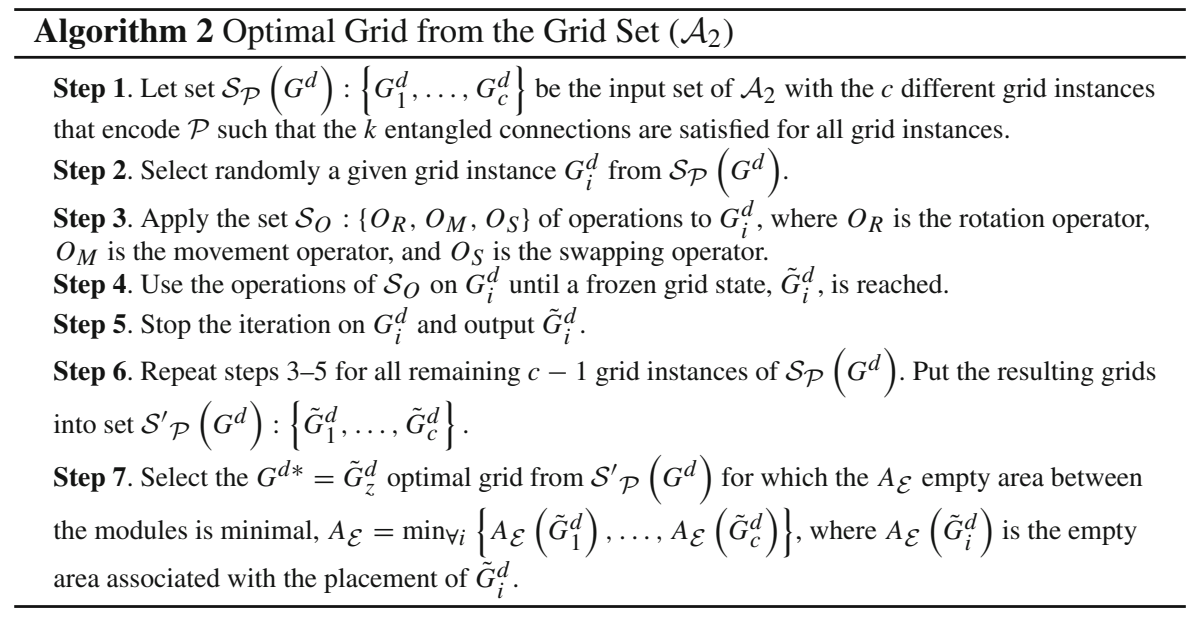

The discussion of algorithm $\mathcal{A}_{2}$ is as follows. After the preliminaries of Steps 1-2, in Step 3, a set of operations $\mathcal{S}_{O}$ [129-133] are used for the construction of a better grid from the current input grid. The application of $O_{R}$ in a module makes it possible to determine a novel solution when a node is inserted into $G_{i}^{d}$. Operation $O_{R}$ can be executed twice at each position. The complexity of $O_{R}$ is $\mathcal{O}\left(O_{R}\right)=\mathcal{O}(1)$. The $O_{M}$ movement operator is applied for the deletion and re-insertion [132,133] of a particular block in the grid; therefore, it can be decomposed as $O_{M}=o_{D}+o_{I}$, where $o_{D}$ is the delete sub-operator and $o_{I}$ is the insert sub-operator. The $o_{D}$ sub-operator of $O_{M}$ can be applied to any unitary block of the grid; thus, the given $U_{s}$ of $G_{i}^{d}$ selected for deletion can have zero, one, or two diagonal connections in the grid. If $U_{s}$ has only one diagonal connection, then $U_{s}$ is removed, and the diagonal connection is placed at the position of the removed node. If $U_{s}$ has two diagonal connections, then the target unitary block is replaced by the left- or right-diagonal connection, and a diagonal connection is moved to the original position of $U_{S}$. The $o_{I}$ sub-operator of $O_{M}$ is used for the addition of new modules into the grid structure [132,133]. A new node can be inserted into a place between two modules (internal position) or into a 
position pointed by a null pointer (external position). The updating process of $O_{M}$ therefore has a complexity $\mathcal{O}\left(O_{M}\right)=\mathcal{O}(1+h)$, where $h$ is the height of the current grid.

The $O_{S}$ swapping operator [132,133] is applied for the swapping of some unitary blocks in the grid structure. Since $O_{S}$ can also be decomposed as $O_{S}=o_{D}+o_{I}$, the complexity of $O_{S}$ is $\mathcal{O}\left(O_{S}\right)=\mathcal{O}(1+h)$.

In Step 4 , the desired grid $\tilde{G}_{i}^{d}$ is achieved from $G_{i}^{d}$ only by application of the $O_{R}, O_{M}, O_{S}$ operations from $\mathcal{S}_{O}$.

In Step 7, as the $G^{d *}$ optimal grid is determined, the optimal placement $\mathcal{P}^{*}$ is trivially yielded by the one-to-one correspondence of $G^{d *}$ and $\mathcal{P}^{*}$. The grid $G^{d *}$ therefore identifies the optimal placement $\mathcal{P}^{*}$ with the $k$ entangled connections between the unitary blocks of the quantum circuit, that concludes the proof.

\subsubsection{Computational complexity}

From the definition of $\mathcal{A}_{2}$, for the selection of the optimal placement from the set of $c$ solutions of $\mathcal{S}_{\mathcal{P}}\left(G^{d}\right)$ at $m$ unitary blocks, the computational complexity of $\mathcal{A}_{2}$ is

$$
\begin{aligned}
\mathcal{O}\left(\mathcal{A}_{2}\right) & =\mathcal{O}\left(c \cdot m\left(O_{R}+O_{M}+O_{S}\right)\right) \\
& =\mathcal{O}\left(c \cdot m\left(O_{R}+2\left(o_{D}+o_{I}\right)\right)\right) .
\end{aligned}
$$

\section{Decoherence dynamics estimation}

In this section, we define a method for the decoherence dynamics estimation of superconducting quantum circuits. In the description, we assume that a particular unitary block $U_{j}$ integrates a number of Josephson junction units $J$ and capacitor units $(C$ is the capacitance of a capacitor); for simplicity, we assume charge qubit circuits [121] in the system model. The unitary blocks formulate a larger unit called a quantum circuit. The quantum circuits are integrated into a multilayer structure with $L$ layers and $K$ columns. A quantum circuit in the $i$ th layer $l_{i}$ and $k$ th column of $G_{Q G}^{L, K}$ is referred to as $C^{l_{i}, k}$. We study a scenario in which the capacitor units of the quantum circuits are interfering with each other [129-131] and reveal the effects of the interference on the distribution of the Gaussian noise in the Josephson energy [121].

\subsection{Capacitor interference in the charge qubit model}

Let $C^{l_{i}, k}$ be a reference charge qubit $\mathcal{C}_{q}$ quantum circuit of the $i$ th layer $l_{i}$ and $k$ th column of $G_{Q G}^{L, K}$. Due to the presence of interference between the capacitor units of the charge qubit circuits, we define the interference coefficients [129-131] $\alpha, \varphi$, and $\Phi$ as follows.

Let $\alpha$ be the area capacitance interference defined between an area of the reference quantum circuit $C^{l_{i}, k}$ and areas of $C^{l_{i-1}, k}$ and $C^{l_{i+1}, k}$. Let coefficient $\varphi$ be the fringe capacitance interference coefficient defined between a side of the reference circuit $C^{l_{i}, k}$ and any parts of $C^{l_{i-1}, k}$ and $C^{l_{i+1}, k}$. Finally, let $\Phi$ be the coupling capacitance 
interference coefficient defined on the same layer $l_{i}$ from a side of the reference circuit $C^{l_{i}, k}$ to a given side $C^{l_{i}, k+1}[129-131]$.

Let us assume further that $C^{l_{i}, k}$ contains $u^{l_{i}, k}$ unitary blocks, $U_{i}, 1 \leq i \leq u^{l_{i}, k}$. Let $A_{i}$ be the area (see (2)) of a particular $U_{i}$. Let us assume that a given $C^{l_{i}, k}$ quantum circuit of the $i$ th layer $l_{i}$, where $k$ identifies the column number in the $G_{Q G}^{L, K}$ multilayer grid structure, contains $u^{l_{i}, k}$ unitary blocks; therefore, the area of $C^{l_{i}, k}$ is

$$
A\left(C^{l_{i}, k}\right)=\sum_{i=1}^{u^{l_{i}, k}} A_{i}=\sum_{i=1}^{u_{i}^{l_{i}, k}} D_{i} N_{i},
$$

where $N_{i}$ (i.e., height of $C^{l_{i}, k}$ ) is given by (1), while $D_{i}$ is the depth (i.e., width of $\left.C^{l_{i}, k}\right)$.

As the capacitor interference is analyzed for a particular $C^{l_{i}, k}$ with nonzero $A\left(C^{l_{i}, k}\right)$, some geometrical considerations also come up because the capacitor interference does not just depend on the amount of overlap between the circuits, but it also differs for the interlayer (quantum circuits from different layers of $G_{Q G}^{L, K}$ ) and intralayer (quantum circuits in the same layer of $G_{Q G}^{L, K}$ ) quantum circuits.

The coefficients $\alpha, \varphi$, and $\Phi$ are illustrated in Fig. 1 for a $G_{Q G}^{L, K}$ multilayer charge qubit $\mathcal{C}_{q}$ quantum circuit structure. These quantities are used for the decoherence estimation in the multilayer charge qubit quantum circuit model.

\subsubsection{Interlayer interference at full overlap}

Let $\bar{A}\left(C^{l_{i}, k}, C^{l_{j}, k}\right)$ be the overlap between the $A\left(C^{l_{i}, k}\right)$ and $A\left(C^{l_{j}, k}\right)$ areas of circuits $C^{l_{i}, k}$ and $C^{l_{j}, k}$ in the layers of $l_{i}$ and $l_{j}$, given as

$$
\bar{A}\left(C^{l_{i}, k}, C^{l_{j}, k}\right)=\sum_{i=1}^{w^{l_{i, j}, k}} D_{i} N_{i},
$$

where $w^{l_{i, j}, k}$ identifies the number of overlapped unitary blocks. If $C^{l_{i}, k}$ is in a full overlap relation with $C^{l_{j}, k}$, then the quantity $\bar{A}\left(C^{l_{i}, k}, C^{l_{j}, k}\right)$ is as

$$
\bar{A}\left(C^{l_{i}, k}, C^{l_{j}, k}\right)=A\left(C^{l_{i}, k}\right)
$$

Let $\alpha\left(\bar{A}\left(C^{l_{i}, k}, C^{l_{j}, k}\right)\right)$ be the area capacitance interference at overlapped area $\bar{A}\left(C^{l_{i}, k}, C^{l_{j}, k}\right)$ between the $A\left(C^{l_{i}, k}\right)$ area of the reference quantum circuit $C^{l_{i}, k}$, and the $A\left(C^{l_{j}, k}\right)$ area of $C^{l_{j}, k}$. Using (13),

$$
\alpha\left(\bar{A}\left(C^{l_{i}, k}, C^{l_{j}, k}\right)\right)=\alpha\left(A\left(C^{l_{i}, k}\right)\right)=\alpha\left(D^{l_{i}, k}\right) N^{l_{i}, k},
$$

where $D^{l_{i}, k}=\sum_{i=1}^{u^{l_{i}, k}} D_{i}$, and $N^{l_{i}, k}=\sum_{i=1}^{u_{i}^{l_{i}, k}} N_{i}$. 


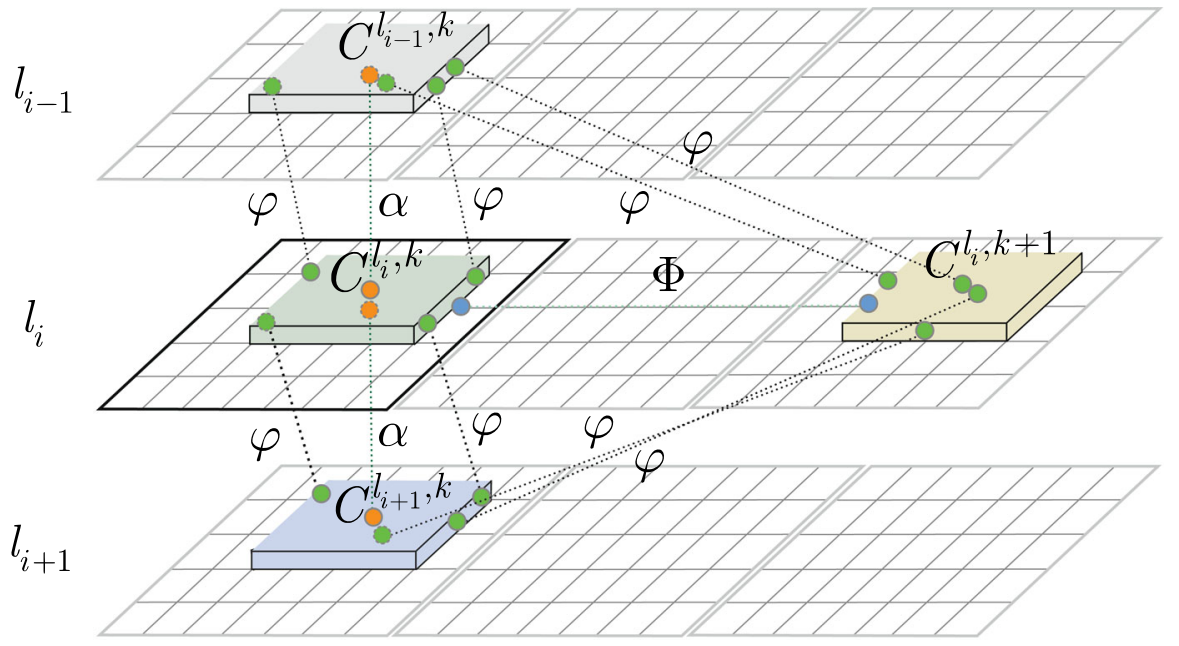

Fig. 1 Representation of the interference coefficients $\alpha, \varphi$, and $\Phi$ with respect to a reference charge qubit quantum circuit $C^{l_{i}, k}$ (depicted in green) for the decoherence estimation in a $G_{Q G}^{L, K}$ multilayer structure $\mathcal{C}_{q}$. Each layer contains several unitary blocks $U_{j}$ with charge qubit circuits. Each $U_{j}$ integrates Josephson junction elements, capacitors, wires, and other elements. The quantum circuit of the $i$ th layer, $l_{i}$, is referred to as $C^{l_{i}, k}$, where $k$ is the column number in the $G_{Q G}^{L, K}$ multilayer grid structure. The capacitors of the charge qubit quantum circuits are interfering with each other, which induces different capacitance coefficients between the quantum circuits. With respect to the $C^{l_{i}, k}$ reference quantum circuit of the $i$ th layer $l_{i}$, the quantity $\alpha$ refers to the area capacitance (between an area of the reference quantum circuit $C^{l_{i}, k}$ and areas of $C^{l_{i-1}, k}$ and $C^{l_{i+1}, k}$ ), the quantity $\varphi$ is the fringe capacitance coefficient (from a side of the reference circuit $C^{l_{i}, k}$ to any parts of $C^{l_{i-1}, k}$ and $C^{l_{i+1}, k}$ ), and $\Phi$ is the coupling capacitance coefficient defined on the same layer (from a side of the reference circuit $C^{l_{i}, k}$ to a side $C^{l_{i}, k+1}$ )

Then, let be the $\varphi\left(d\left(C^{l_{i}, k}, C^{l_{j}, k}\right)\right)$ capacitance interference coefficient defined between the sides of the reference circuit $C^{l_{i}, k}$ and the area of $C^{l_{j}, k}$, where $d\left(C^{l_{i}, k}, C^{l_{j}, k}\right)$ is the vertical distance between circuits $C^{l_{i}, k}$ and $C^{l_{j}, k}$. Since

$$
d\left(C^{l_{i}, k}, C^{l_{j}, k}\right)=L 2\left(l_{i}, l_{j}\right),
$$

where $L 2\left(l_{i}, l_{j}\right)=\left|x_{i}-x_{j}\right|+\left|y_{i}-y_{j}\right|+\left|z_{i}-z_{j}\right| f_{c}$ is the distance function between the layers $l_{i}, l_{j}$ in $G_{Q G}^{L, K}$, where $f_{c}(\cdot)$ is a cost function between the layers, thus the quantity $\varphi\left(d\left(C^{l_{i}, \hat{k}}, C^{l_{j}, k}\right)\right)$ can be rewritten as

$$
\varphi\left(d\left(C^{l_{i}, k}, C^{l_{j}, k}\right)\right)=\varphi\left(L 2\left(l_{i}, l_{j}\right)\right) .
$$

Using (14), (15) and (16), the $\mathcal{D}_{l_{i}, l_{j}}^{A\left(C_{i}^{l_{i}, k}\right)}\left(C^{l_{i}, k}, C^{l_{j}, k}\right)$ interlayer interference at full overlapping circuits $C^{l_{i}, k}$ and $C^{l_{j}, k}$ between layers $l_{i}, l_{j}$ is defined as

$$
\mathcal{D}_{l_{i}, l_{j}}^{A\left(C_{i}^{l_{i}, k}\right)}\left(C^{l_{i}, k}, C^{l_{j}, k}\right)=\alpha\left(D^{l_{i}, k}\right) N^{l_{i}, k}+2 \varphi\left(L 2\left(l_{i}, l_{j}\right)\right) N^{l_{i}, k} .
$$




\subsubsection{Interlayer interference at partial or zero overlap}

If the circuits $C^{l_{i}, k}$ and $C^{l_{j}, k}$ in the layers of $l_{i}$ and $l_{j}$ have a partial or zero overlap, then

$$
\bar{A}\left(C^{l_{i}, k}, C^{l_{j}, k}\right)<A\left(C^{l_{i}, k}\right),
$$

and

$$
\alpha\left(\bar{A}\left(C^{l_{i}, k}, C^{l_{j}, k}\right)\right)=\alpha\left(\bar{D}^{l_{i}, k}\right) \bar{N}^{l_{i}, k}<\alpha\left(D^{l_{i}, k}\right) N^{l_{i}, k}
$$

where $\bar{D}^{l_{i}, k}=\sum_{i=1}^{o_{i}^{l_{i}, k}} D_{i}$ (overlap width), and $\bar{N}^{l_{i}, k}=\sum_{i=1}^{o_{i}, k} N_{i}$ (overlap length), where $o^{l_{i}, k}$ identifies the amount of overlap of $C^{l_{i}, k}, o^{l_{i}, k}<u^{l_{i}, k}$.

By similar assumptions, the $\mathcal{D}_{l_{i}, l_{j}}^{\bar{A}\left(C^{l_{i}, k}, C^{l_{j}, k}\right)}\left(C^{l_{i}, k}, C^{l_{j}, k}\right)$ interlayer interference at partial overlapping circuits $C^{l_{i}, k}$ and $C^{l_{j}, k}$ between two layers $l_{i}, l_{j}$ is defined as

$$
\begin{aligned}
& \mathcal{D}_{l_{i}, l_{j}}^{\bar{A}\left(C^{l_{i}, k}, C^{l_{j}, k}\right)}\left(C^{l_{i}, k}, C^{l_{j}, k}\right) \\
& \quad=\alpha\left(\bar{D}^{l_{i}, k}\right) \bar{N}^{l_{i}, k}+2 \varphi\left(\mathrm{L} 2\left(l_{i}, l_{j}\right)\right) \bar{N}^{l_{i}, k} .
\end{aligned}
$$

As the overlap between $C^{l_{i}, k}$ and $C^{l_{j}, k}$ is zero, $\bar{A}\left(C^{l_{i}, k}, C^{l_{j}, k}\right)=0$, then

$$
\mathcal{D}_{l_{i}, l_{j}}^{0}\left(C^{l_{i}, k}, C^{l_{j}, k}\right)=2 \varphi\left(h\left(C^{l_{i}, k}, C^{l_{j}, k}\right)\right) N^{l_{i}, k} \varsigma,
$$

where $h\left(C^{l_{i}, k}, C^{l_{j}, k}\right)$ is the horizontal distance between $C^{l_{i}, k}$ and $C^{l_{j}, k}$, while $\varsigma$ is a constant (decay factor [129-131]).

\subsubsection{Intralayer interference}

Let $h\left(C^{l_{i}, k}, C^{l_{i}, f}\right)$ be the horizontal distance between two parallel circuits $C^{l_{i}, k}$ and $C^{l_{i}, f}$ in the same layer $l_{i}$. Then, $\bar{A}\left(C^{l_{i}, k}, C^{l_{i}, f}\right)=0$, and the intralayer interference is between $C^{l_{i}, k}$ and $C^{l_{i}, f}$

$$
\mathcal{D}_{l_{i}, l_{j}}^{0}\left(C^{l_{i}, k}, C^{l_{i}, f}\right)=\Phi\left(h\left(C^{l_{i}, k}, C^{l_{j}, k}\right)\right) N^{l_{i}, k}
$$

where $\Phi\left(h\left(C^{l_{i}, k}, C^{l_{j}, k}\right)\right)$ is coupling capacitance coefficient.

The same result holds for two perpendicular circuits $C^{l_{i}, k}$ and $C^{l_{i}, f}$ in the same layer $l_{i}$ with $v\left(C^{l_{i}, k}, C^{l_{j}, k}\right)$ vertical distance, as

$$
\mathcal{D}_{l_{i}, l_{j}}^{0}\left(C^{l_{i}, k}, C^{l_{i}, f}\right)=\Phi\left(v\left(C^{l_{i}, k}, C^{l_{j}, k}\right)\right) N^{l_{i}, k} .
$$




\subsubsection{Capacitor interference estimation}

For the determination of quantities (17), (20) and (22), the $\mathcal{A}_{3}$ interference estimation algorithm is defined. The algorithm also finds the coefficients $\alpha\left(U_{j}\right), \varphi\left(U_{j}\right)$ and $\Phi\left(U_{j}\right)$ of a particular unit $U_{j}$. The steps of $\mathcal{A}_{3}$ are detailed in Algorithm 3 .

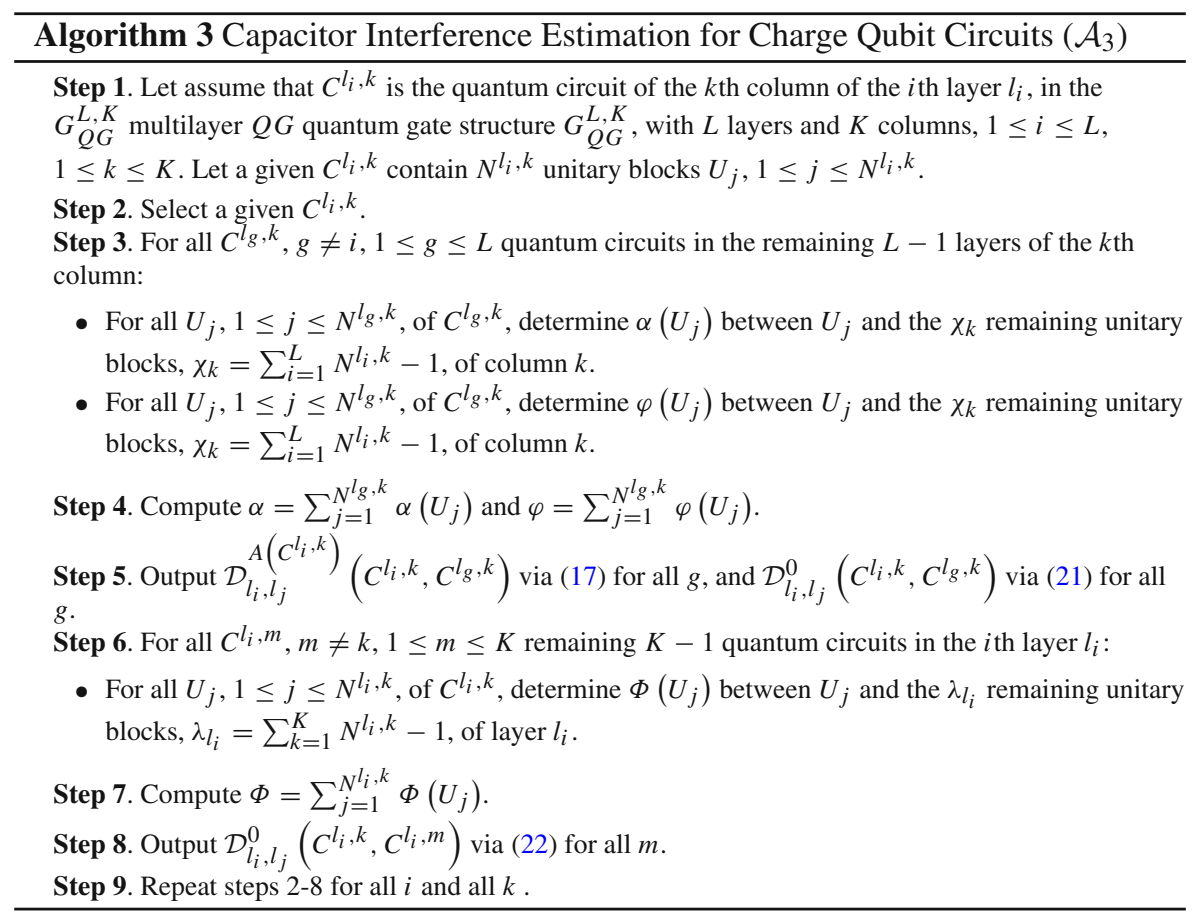

The result on the effect of interference coefficients $\alpha\left(U_{j}\right), \varphi\left(U_{j}\right), \Phi\left(U_{j}\right)$ of $U_{j}$ on the distribution of the decoherence of $U_{j}$ is concluded in Theorem 2.

\subsubsection{Decoherence distribution}

Theorem 2 For a $\mathcal{C}_{q}: U_{j}$ charge qubit structure with a Gaussian noise $\mathcal{D}\left(U_{j}\right)$, any nonzero $\Omega\left(U_{j}\right)$, where $\Omega\left(U_{j}\right)=\alpha\left(U_{j}\right)+\varphi\left(U_{j}\right)+\Phi\left(U_{j}\right)$, modifies the noise distribution $\mathcal{D}\left(U_{j}\right) \in \mathcal{N}\left(\bar{E}_{J}, \sigma_{E_{J}}^{2}\right)$ of the Josephson energy $E_{J}$ of $U_{j}$, where $\bar{E}_{J}$ is the mean, and $\sigma_{E_{J}}^{2}$ is the variance.

Proof Let assume that the quantum circuits in $G_{Q G}^{L, K}$ multilayer grid are implemented by $\mathcal{C}_{q}$ charge qubit superconducting qubit structures. Each $\mathcal{C}_{q}$ is equipped with a capacitor with capacitance $C$, and Josephson junction $J$ with energy $E_{J}$ that is biased by a voltage $U[121]$. Let $U_{j}$ be a reference $\mathcal{C}_{q}$ in the $G_{Q G}^{L, K}$ multilayer quantum circuit structure. 
To describe the interactions and the evolution of a superconducting charge qubit circuit, we utilize the circuit Hamiltonian.

Let $n$ be the number of excess Cooper-pairs on the island, then the $H\left(\mathcal{C}_{q}\right)$ circuit Hamiltonian of $\mathcal{C}_{q}$ is as [63]

$$
H\left(\mathcal{C}_{q}\right)=4 E_{C}\left(n-n_{g}\right)^{2}-E_{J} \cos \phi
$$

where $E_{C}$ is the charging energy, $E_{J}$ is the Josephson energy, and $n_{g}$ is a control parameter (effective offset charge), as

$$
n_{g}=-\frac{C V_{0}}{2 e},
$$

where $V_{0}$ is a gate voltage, while $\phi$ is the superconducting wave function phase difference across $J$ (a $2 \pi$-periodic operator of the phase difference). Note, the operators satisfy the commutation relation [63],

$$
[\phi, n]=i \text {. }
$$

Then, applying a voltage that yields $n_{g}=1 / 2$ for the period $t_{0}<t<t_{0}+\Delta t$, the $|\psi\rangle$ system state of $U_{j}$ will oscillate between states $|0\rangle$ and $|1\rangle$. Therefore, $|\psi(t)\rangle$ is as [121]

$$
|\psi(t)\rangle=\exp \left(-\frac{i}{\hbar} \hat{T} \int_{t_{0}}^{t} \hat{H}\left(t^{\prime}\right) \mathrm{d} t^{\prime}\right)\left|\psi\left(t_{0}\right)\right\rangle,
$$

where $\hat{H}$ is a Hamiltonian, $\hat{T}$ is the time-ordering operator, and $\hbar$ is the reduced Planck constant. If the Hamiltonian is constant, then (27) can be rewritten as

$$
|\psi(t)\rangle=e^{-\frac{i}{\hbar} \hat{H}\left(t-t_{0}\right)}\left|\psi\left(t_{0}\right)\right\rangle
$$

Using the eigenstates $1 / \sqrt{2}\left(\begin{array}{l}1 \\ 1\end{array}\right), 1 / \sqrt{2}\left(\begin{array}{l}1 \\ -1\end{array}\right)$ at $n_{g}=1 / 2,|\psi(t)\rangle$ can be rewritten as [121]

$$
\begin{array}{rc}
|\psi(t)\rangle \quad & =e^{-\frac{i}{\hbar} \hat{H}\left(t-t_{0}\right)}\left(\left(\begin{array}{l}
1 \\
1
\end{array}\right)-\left(\begin{array}{c}
1 \\
-1
\end{array}\right)\right) \\
= & e^{+\frac{i}{\hbar} \frac{E_{J}}{2}\left(t-t_{0}\right)}\left(\begin{array}{l}
1 \\
1
\end{array}\right)-e^{-\frac{i}{\hbar} \frac{E_{J}}{2}\left(t-t_{0}\right)}\left(\begin{array}{c}
1 \\
-1
\end{array}\right),
\end{array}
$$

therefore $|\psi(t)\rangle$ is yielded from (29) as

$$
|\psi(t)\rangle=\left(\begin{array}{l}
e^{+\frac{i}{\hbar} \frac{E_{J}}{2}\left(t-t_{0}\right)}-e^{-\frac{i}{\hbar} \frac{E_{J}}{2}\left(t-t_{0}\right)} \\
e^{+\frac{i}{\hbar} \frac{E_{J}}{2}\left(t-t_{0}\right)}+e^{-\frac{i}{\hbar} \frac{E_{J}}{2}\left(t-t_{0}\right)}
\end{array}\right) .
$$

The $\operatorname{Pr}(|\psi(t)\rangle=|0\rangle)$ probability at $t=t_{0}+\Delta t$ is therefore as

$$
\operatorname{Pr}\left(\left|\psi\left(t_{0}+\Delta t\right)\right\rangle=|0\rangle\right)=\left|\left\langle 0 \mid \psi\left(t_{0}+\Delta t\right)\right\rangle\right|^{2}=\cos ^{2}\left(E_{J} / 2 \hbar \Delta t\right)
$$


and $\operatorname{Pr}(|\psi(t)\rangle=|1\rangle)$ at $t=t_{0}+\Delta t$ is as

$$
\operatorname{Pr}\left(\left|\psi\left(t_{0}+\Delta t\right)\right\rangle=|1\rangle\right)=\left|\left\langle 1 \mid \psi\left(t_{0}+\Delta t\right)\right\rangle\right|^{2}=\sin ^{2}\left(E_{J} / 2 \hbar \Delta t\right) .
$$

The $\operatorname{Pr}\left(\left|\psi\left(t_{0}+\Delta t\right)\right\rangle=|0\rangle\right)$ and $\operatorname{Pr}\left(\left|\psi\left(t_{0}+\Delta t\right)\right\rangle=|1\rangle\right)$ probabilities are therefore oscillated with an angular frequency $f_{0}$ as

$$
f_{0}=\frac{E_{J}}{\hbar}
$$

As one can conclude, setting $\Delta t$ to $\Delta t=\pi \hbar / 2 E_{J}$ yields the equal superposition of the charge states.

Let assume that the $\mathcal{D}\left(U_{j}\right)$ decoherence of the Josephson energy $E_{J}$ of $U_{j}$ is a Gaussian noise [121]

$$
\mathcal{D}\left(U_{j}\right) \in \mathcal{N}\left(\bar{E}_{J}, \sigma_{E_{J}}^{2}\right),
$$

with mean $\bar{E}_{J}$ and variance $\sigma_{E_{J}}^{2}$, with standard deviation $\sigma_{E_{J}}$ as

$$
\sigma_{E_{J}}=\Delta E_{J}=\left|E_{J}^{(0)}-E_{J}^{(1)}\right|
$$

where $\Delta E_{J}$ is the width of the Gaussian distribution of $E_{J}$ centered on $\bar{E}_{J}$, and $E_{J}^{(0)}$ and $E_{J}^{(1)}$ are parameterize the width $\Delta E_{j}$.

Thus, in the presence of (34), the $\operatorname{Pr}(|\psi(t)\rangle=|1\rangle)$ probability in (32) is yielded as [121]

$$
\begin{aligned}
& \operatorname{Pr}_{\mathcal{D}\left(U_{j}\right)}\left(\left|\psi\left(t_{0}+\Delta t\right)\right\rangle=|1\rangle\right) \\
& =1 / \sigma_{E_{J}} \sqrt{2 \pi} \int_{-\infty}^{\infty} e^{-\frac{\left(E_{J}-\bar{E}_{J}\right)^{2}}{2 \sigma_{E_{J}}^{2}}} \sin ^{2}\left(E_{J} / 2 \hbar \Delta t\right) d E_{J},
\end{aligned}
$$

where $E_{J}$ is a Gaussian random variable,

$$
E_{J} \in \mathcal{N}\left(\bar{E}_{J}, \sigma_{E_{j}}^{2}\right)
$$

The probability in (36) can be rewritten as

$$
\begin{aligned}
& \operatorname{Pr}_{\mathcal{D}\left(U_{j}\right)}\left(\left|\psi\left(t_{0}+\Delta t\right)\right\rangle=|1\rangle\right) \\
& =1 / 2\left(1-e^{-\left(\frac{\Delta t\left(\sigma_{E_{J}}\right)}{\hbar}\right)^{2}} \cos \left(\bar{E}_{J} / \hbar \Delta t\right)\right) \\
& =1 / 2\left(1-e^{-(\Delta t \zeta)^{2}} \cos \left(\bar{E}_{J} / \hbar \Delta t\right)\right)
\end{aligned}
$$


thus in the presence of a Gaussian noise $\mathcal{D}\left(U_{j}\right)$ the system oscillates with frequency $f=\bar{E}_{J} / \hbar$, and it decreases exponentially with a time constant [121]

$$
\zeta=\frac{\sigma_{E_{J}}}{\hbar}
$$

where $\sigma_{E_{J}}$ is the standard deviation of $\mathcal{D}\left(U_{j}\right)$ in the Josephson energy [121].

The decoherence associated with the capacitance interference is derived as follows. Let us evaluate the effect of a nonzero $\Omega\left(U_{j}\right)$, where $\Omega\left(U_{j}\right)$ is the sum of all capacitance interference coefficients associated with $U_{j}$ as

$$
\Omega\left(U_{j}\right)=\alpha\left(U_{j}\right)+\varphi\left(U_{j}\right)+\Phi\left(U_{j}\right)
$$

where the coefficients $\alpha\left(U_{j}\right) \geq 0, \varphi\left(U_{j}\right) \geq 0$ and $\Phi\left(U_{j}\right) \geq 0$ are determined for a particular $U_{j}$ via Algorithm 3 .

At any nonzero $\Omega\left(U_{j}\right)$, the capacitance $C$ of $U_{j}$ can be rewritten as $C^{\prime}$ as

$$
C^{\prime}=C+\Omega\left(U_{j}\right)
$$

and the resulting $n_{g}^{\prime}$ is as

$$
n_{g}^{\prime}=-\frac{C^{\prime} V_{0}}{2 e}=-\frac{\left(C+\Omega\left(U_{j}\right)\right) V_{0}}{2 e} .
$$

Using (42), the Josephson energy $E_{J}^{\prime}$ at a nonzero $\Omega\left(U_{j}\right)$ is as

$$
E_{J}^{\prime}=\frac{4 E_{C}^{\prime}\left(n-n_{g}^{\prime}\right)^{2}-H^{\prime}\left(\mathcal{C}_{q}\right)}{\cos \phi},
$$

which is a Gaussian random variable

$$
E_{J}^{\prime} \in \mathcal{N}\left(\bar{E}_{J}^{\prime}, \sigma_{E_{J}^{\prime}}^{2}\right)
$$

with mean $\bar{E}_{J}^{\prime}$ and standard deviation $\sigma_{E_{J}^{\prime}}$

$$
\sigma_{E_{J}^{\prime}}=\Delta E_{J}^{\prime}=\left|E_{J}^{\prime(0)}-E_{J}^{\prime(1)}\right|=\sigma_{E_{J}^{\prime}}+\sigma_{\Omega}
$$

where $\Delta E_{J}^{\prime}$ is the width of the Gaussian distribution of $E_{J}^{\prime}$ centered on $\bar{E}_{J}^{\prime}$, while $E_{J}^{\prime(0)}$ and $E_{J}^{\prime(1)}$ parameterize the width of $\Delta E_{J}^{\prime}$.

Thus, $\sigma_{\Omega}$ is as

$$
\sigma_{\Omega}=\|\left|E_{J}^{(0)}-E_{J}^{(1)}\right|-\left|E_{J}^{(0)}-E_{J}^{(1)}\right| \mid,
$$


while the Hamiltonian $H^{\prime}\left(\mathcal{C}_{q}\right)$ in (43) is as,

$$
H^{\prime}\left(\mathcal{C}_{q}\right)=4 E_{C}^{\prime}\left(n-n_{g}^{\prime}\right)^{2}-E_{J}^{\prime} \cos \phi
$$

Therefore, the noise evolution of $U_{j}$ at the degeneracy point $n_{g}^{\prime}=1 / 2$ in the presence of $\Omega\left(U_{j}\right)>0$ is as

$$
\begin{aligned}
& \operatorname{Pr}_{\mathcal{D}\left(U_{j}\right)>0}^{\Omega\left(U_{j}\right)>0}\left(\left|\psi\left(t_{0}+\Delta t\right)\right\rangle=|1\rangle\right) \\
& =1 / \sigma_{E_{J}^{\prime}} \sqrt{2 \pi} \int_{-\infty}^{\infty} e^{-\frac{\left(E_{J}^{\prime}-\bar{E}_{J}^{\prime}\right)^{2}}{2 \sigma_{E_{J}^{\prime}}^{2}}} \sin ^{2}\left(E_{J}^{\prime} / 2 \hbar \Delta t\right) d E_{J}^{\prime},
\end{aligned}
$$

that can be rewritten as

$$
\begin{aligned}
& \operatorname{Pr}_{\mathcal{D}\left(U_{j}\right)}^{\Omega\left(U_{j}\right)>0}\left(\left|\psi\left(t_{0}+\Delta t\right)\right\rangle=|1\rangle\right) \\
& \left.=1 / 2\left(1-e^{-\left(\frac{\Delta t \sigma}{\hbar} E_{J}^{\prime}\right.}\right)^{2} \cos \left(\bar{E}_{J}^{\prime} / \hbar \Delta t\right)\right) \\
& =1 / 2\left(1-e^{-\left(\Delta t \zeta^{\prime}\right)^{2}} \cos \left(\bar{E}_{J}^{\prime} / \hbar \Delta t\right)\right)
\end{aligned}
$$

where

$$
\zeta^{\prime}=\frac{\sigma_{E_{J}^{\prime}}}{\hbar}=\frac{\sigma_{E_{j}}+\sigma_{\Omega}}{\hbar}=\frac{\Delta E_{J}^{\prime}}{\hbar} .
$$

Thus, in the presence of a $\Omega\left(U_{j}\right)>0$, the noisy system oscillates with frequency

$$
f^{\prime}=\frac{\bar{E}_{J}^{\prime}}{\hbar},
$$

and it decreases exponentially with a time constant as given in (50).

Therefore, at a nonzero $\Omega\left(U_{j}\right)$, the distribution of the $\mathcal{D}\left(U_{j}\right)_{\Omega\left(U_{j}\right)}$ Gaussian noise of $U_{j}$ is yielded as

$$
\mathcal{D}\left(U_{j}\right)_{\Omega\left(U_{j}\right)} \in \mathcal{N}\left(\bar{E}_{J}^{\prime}, \Delta E_{J}^{\prime}\right)=\mathcal{N}\left(\bar{E}_{J}^{\prime}, \sigma_{E_{J}}^{2}+\sigma_{\Omega}^{2}\right)
$$

that concludes the proof.

By assuming charge qubit structure in all computational units of circuits of the $Q G$ quantum circuit, the results of Theorem 2 can be extended, and the effects of the interlayer interference between full (see (17)) and partial overlapping (see (21)) circuits $C^{l_{i}, k}$ and $C^{l_{j}, k}$ between layers $l_{i}, l_{j}$, and the intralayer interference (see (22)) between two circuits $C^{l_{i}, k}$ and $C^{l_{i}, f}$ in the same layer can also be yielded. 


\subsection{Decoherence estimation method}

The decoherence estimation method $\mathcal{A}_{4}$ is defined in Algorithm 4. From the output of $\mathcal{A}_{4}$, the $\mathcal{P}^{*}$ optimal placement can be re-determined by algorithms $\mathcal{A}_{1}$ and $\mathcal{A}_{2}$, by adding the constraint of the minimization of the corresponding quantities $\Delta=$ $\left|\bar{E}_{J}^{\prime}-\bar{E}_{J}\right|$ and $\gamma=\left|\Delta E_{J}^{\prime}-\Delta E_{J}\right|$.

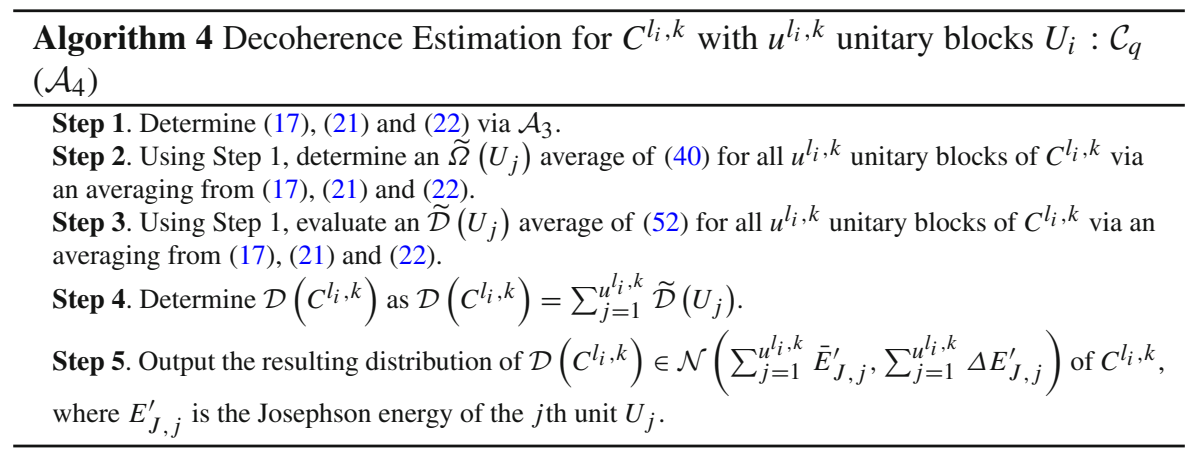

\subsection{NISQ applications}

The proposed results can be applied in experimental NISQ [2] settings. The system model particularly focuses on near-term superconducting charge qubit architectures; however, the placement algorithm can be extended to other experimental scenarios such as trapped ion circuits. The decoherence estimation method is directly applicable in superconducting qubit architectures, NISQ algorithm implementations, and in the realization of quantum error correction using superconducting qubits [63]. Another near-term application is in NISQ qubit gate-model quantum computer architectures with a large number of qubits and quantum gates in the physical layer $[5,10,11,136]$. As a future work, our aim is to analyze the performance of the system model in these extended NISQ scenarios.

\section{Conclusions}

Here, we defined a method for layout generation and decoherence estimation in superconducting gate-model quantum computers. The optimal physical layout was determined by an iterative procedure that utilizes our grid structure to encode the various placements of the quantum circuit. We set a multilayer grid structure for the analysis of decoherence distribution in the presence of different capacitor interferences between the quantum circuits. The results can be used in superconducting gate-model quantum computers and in the near-term quantum devices of the quantum Internet. 
Acknowledgements The research reported in this paper has been supported by the Hungarian Academy of Sciences (MTA Premium Postdoctoral Research Program 2019), by the National Research, Development and Innovation Fund (TUDFO/51757/2019-ITM, Thematic Excellence Program), by the National Research Development and Innovation Office of Hungary (Project No. 2017-1.2.1-NKP-2017-00001), by the Hungarian Scientific Research Fund - OTKA K-112125 and in part by the BME Artificial Intelligence FIKP Grant of EMMI (Budapest University of Technology, BME FIKP-MI/SC).

Author contributions L. GY. designed the protocol, wrote the manuscript and analyzed the results.

Funding Open access funding provided by Budapest University of Technology and Economics.

Data Availability Statement This work does not have any experimental data.

\section{Compliance with ethical standards}

Conflict of interest There are no competing interests.

Competing non-financial interests statement There are no competing non-financial interests.

Competing financial interests statement There are no competing financial interests.

Ethical standard This work did not involve any active collection of human data.

Open Access This article is licensed under a Creative Commons Attribution 4.0 International License, which permits use, sharing, adaptation, distribution and reproduction in any medium or format, as long as you give appropriate credit to the original author(s) and the source, provide a link to the Creative Commons licence, and indicate if changes were made. The images or other third party material in this article are included in the article's Creative Commons licence, unless indicated otherwise in a credit line to the material. If material is not included in the article's Creative Commons licence and your intended use is not permitted by statutory regulation or exceeds the permitted use, you will need to obtain permission directly from the copyright holder. To view a copy of this licence, visit http://creativecommons.org/licenses/by/4.0/.

\section{A Appendix}

\section{A.1 Placement of unitary blocks}

The $G^{d}$ grid structure, $d=2, N=4$, and the yielding placement $\mathcal{P}$ of the unitary blocks with the defined entangled connections are depicted in Fig. 2. 


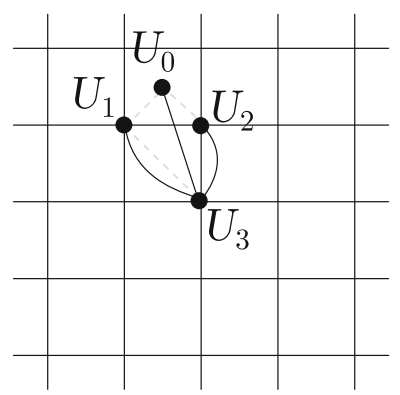

(a)

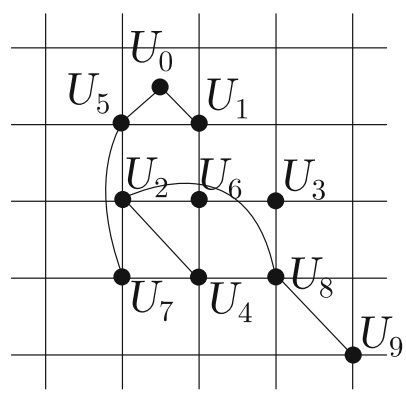

(c)

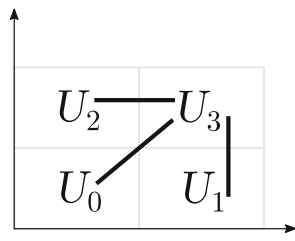

(b)

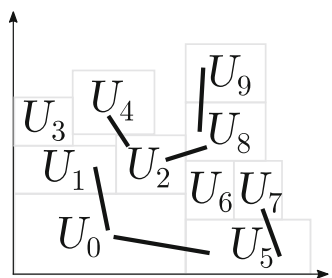

(d)

Fig. $2 G^{2}$ grid structure of a placement $\mathcal{P}$ of the unitary blocks with a set $E$ of entangled connections (depicted by solid lines) between the $U_{i}$ blocks. In the grid structure, the first unitary block $U_{0}$ is in the center of a square to identify the bottom-left block in the $\mathcal{P}$ physical layer topology. From a given $U_{i}$, one left and one right unitary block can be evaluated by auxiliary diagonal lines, such that the $U_{i}, i>0$, evaluated blocks are placed in the intersections of the grid. A left-diagonal line represents a right-adjacent block in the physical layer structure, while a right-diagonal line represents an up-adjacent block. The entanglement between the blocks is depicted by the solid lines. a: The first block $U_{0}$ is evaluated into $U_{1}$ and $U_{2}$. From $U_{1}$, a new block, $U_{3}$ is evaluated via the diagonal line (topology relations depicted by gray dashed lines). b: The physical layer topology of the unitary computational blocks extracted from the grid structure of (a) (entanglement between the blocks is depicted by the thick solid lines). c: A grid structure of nine unitary blocks (gray dashed lines for the topology relations are not depicted). d: The physical layer topology of the unitary computational blocks is extracted from the grid structure of (c) (entanglement between the blocks is depicted by the thick solid lines)

\section{A.2 Notations}

The notations of the manuscript are summarized in Table 1. 
Table 1 Summary of notations

\begin{tabular}{|c|c|}
\hline Notation & Description \\
\hline$G^{d}$ & $\begin{array}{l}\text { A } d \text {-dimensional, } N \text { size grid } G^{d} \text { to encode the physical placement } \\
\text { of the } m \text { unitary computational blocks. }\end{array}$ \\
\hline$d$ & Dimension of the grid. \\
\hline$H$ & Hamiltonian operator. \\
\hline$Q G$ & Quantum circuit (structure of quantum gates). \\
\hline$U_{i}$ & $\begin{array}{l}\text { An } i \text { th unitary computational block, it contains a block of unitary } \\
\text { operators. }\end{array}$ \\
\hline$m$ & The number of unitary blocks in the physical layout. \\
\hline$D_{i}$ & Circuit depth of $U_{i}$. \\
\hline$N_{i}$ & $\begin{array}{c}\text { Number of quantum wires, } N_{i}=\max \left(n_{i}^{i n}, n_{i}^{\text {out }}\right), \text { where } n_{i}^{i n} \text { and } \\
n_{i}^{\text {out }} \text { are the number of input and output quantum wires of } U_{i} \text {. }\end{array}$ \\
\hline$A_{i}$ & $\begin{array}{l}\text { Area of } U_{i}, A_{i}=D_{i} N_{i} \\
\text { Physical placement of the } m \text { unitary computational blocks. }\end{array}$ \\
\hline $\mathcal{P}^{*}$ & Optimal placement in the physical layer. \\
\hline$\kappa\left(\mathcal{P}^{*}\right)$ & Minimum bounding rectangle of $\mathcal{P}^{*}$. \\
\hline$\ell$ & $\begin{array}{l}\text { Total length of quantum wires of } \mathcal{P}^{*} \\
\ell=\sum_{i=1}^{m} w_{i}=\min \beta\left(\mathcal{P}^{*}\right) \text {, where } w_{i} \text { is the quantum wire } \\
\text { length associated with } U_{i}, \beta\left(\mathcal{P}^{*}\right) \text { is the sum of the half-bounding } \\
\text { box of interconnections between the unitary blocks. }\end{array}$ \\
\hline$U_{0}$ & $\begin{array}{l}\text { A unitary block that identify a unique base bottom-left coordinate } \\
\text { in } \mathcal{P}^{*} \text {. }\end{array}$ \\
\hline$E$ & A set of $k$ entangled connections, $E=\left\{E_{1}, E_{2}, \ldots, E_{k}\right\}$ \\
\hline $\mathcal{S}_{r}$ & Set of unitary blocks placed adjacent and right to $U_{0}$. \\
\hline $\mathcal{S}_{a}$ & Set of unitary blocks above $U_{0}$. \\
\hline$G^{d *}$ & Optimal grid that encodes $\mathcal{P}^{*}$. \\
\hline $\mathcal{A}_{1}, \mathcal{A}_{2}, \mathcal{A}_{3}, \mathcal{A}_{4}$ & Algorithms of the framework. \\
\hline $\mathcal{S}\left(G^{d}\right)$ & $\begin{array}{l}\text { A set of } c \text { different grid instances that encode a placement such that } \\
\text { the } k \text { entangled connections are satisfied in the physical layout } \\
\mathcal{S}\left(G^{d}\right):\left\{G_{1}^{d}, \ldots, G_{c}^{d}\right\} \text {. }\end{array}$ \\
\hline$c$ & Number of solutions in $\mathcal{S}\left(G^{d}\right)$ \\
\hline$L_{1}$ & List of the $m-1$ unitary blocks ordered by the $y$-coordinates. \\
\hline $\mathcal{S}_{O}$ & $\begin{array}{l}\text { Set of operations, } \mathcal{S}_{O}:\left\{O_{R}, O_{M}, O_{S}\right\} \text {, where } O_{R} \text { is the rotation } \\
\text { operator, } O_{M} \text { is the movement operator, and } O_{S} \text { is the swapping } \\
\text { operator. } O_{M}=o_{D}+o_{I}, O_{S}=o_{D}+o_{I} \text {, where } o_{D} \text { is the delete } \\
\text { sub-operation, and } o_{I} \text { is the insert sub-operator. }\end{array}$ \\
\hline$\tilde{G}_{i}^{d}$ & Frozen grid state. \\
\hline
\end{tabular}


Table 1 continued

\begin{tabular}{|c|c|}
\hline Notation & Description \\
\hline $\mathcal{S}^{\prime}\left(G^{d}\right)$ & Auxiliary set of grids. \\
\hline$A_{\mathcal{E}}$ & $\begin{array}{l}\text { Empty area between the modules, } \\
\qquad A_{\mathcal{E}}=\min _{\forall i}\left\{A_{\mathcal{E}}\left(\tilde{G}_{1}^{d}\right), \ldots, A_{\mathcal{E}}\left(\tilde{G}_{c}^{d}\right)\right\} \text {, where } A_{\mathcal{E}}\left(\tilde{G}_{i}^{d}\right) \text { is the } \\
\text { empty area associated with the placement of } \tilde{G}_{i}^{d} \text {. }\end{array}$ \\
\hline $\mathcal{C}_{q}$ & Charge qubit model. \\
\hline$J$ & Josephson junction in $\mathcal{C}_{q}$. \\
\hline$E_{J}$ & Energy of Josephson junction $J$. \\
\hline$C$ & Capacitance of a capacitor in $\mathcal{C}_{q}$. \\
\hline$G_{Q G}^{L, K}$ & $\begin{array}{l}\text { Multilayer structure of } \mathcal{C}_{q} \text { quantum circuits, with } L \text { layers and } K \\
\text { columns. }\end{array}$ \\
\hline$C^{l_{i}, k}$ & Quantum circuit in the $i$ th layer $l_{i}$, and $k$ th column of $G_{Q G}^{L, K}$. \\
\hline$\alpha, \varphi, \Phi$ & $\begin{array}{l}\text { Interference coefficients, } \alpha \text { is the area interference capacitance, } \varphi \text { is } \\
\text { the fringe interference capacitance coefficient, } \Phi \text { is the coupling } \\
\text { interference capacitance coefficient. }\end{array}$ \\
\hline$u^{l_{i}, k}$ & Number of unitary blocks of $C^{l_{i}, k}, U_{i}, 1 \leq i \leq u^{l_{i}, k}$. \\
\hline$A\left(C^{l_{i}, k}\right)$ & Area of $C^{l_{i}, k}$. \\
\hline$N_{i}$ & Circuit height of $C^{l_{i}, k}$. \\
\hline$D_{i}$ & Circuit depth of $C^{l_{i}, k}$. \\
\hline $\bar{A}\left(C^{l_{i}, k}, C^{l_{j}, k}\right)$ & $\begin{array}{l}\text { Overlap between the } A\left(C^{l_{i}, k}\right) \text { and } A\left(C^{l_{j}, k}\right) \text { areas of circuits } \\
C^{l_{i}, k} \text { and } C^{l_{j}, k} \text {, in the layers of } l_{i} \text { and } l_{j} \text {. }\end{array}$ \\
\hline$\alpha\left(\bar{A}\left(C^{l_{i}, k}, C^{l_{j}, k}\right)\right)$ & $\begin{array}{c}\text { Area interference capacitance at overlapped area } \bar{A}\left(C^{l_{i}, k}, C^{l_{j}, k}\right) \\
\text { between the } A\left(C^{l_{i}, k}\right) \text { area of the reference quantum circuit } \\
C^{l_{i}, k} \text {, and the } A\left(C^{l_{j}, k}\right) \text { area of } C^{l_{j}, k} \text {. }\end{array}$ \\
\hline$\varphi\left(d\left(C^{l_{i}, k}, C^{l_{j}, k}\right)\right)$ & $\begin{array}{l}\text { Capacitance interference coefficient defined between the sides of } \\
\text { the reference circuit } C^{l_{i}, k} \text { and the area of } C^{l_{j}, k} \text {, where } \\
d\left(C^{l_{i}, k}, C^{l_{j}, k}\right) \text { is the vertical distance between circuits } C^{l_{i}, k} \\
\text { and } C^{l_{j}, k} \text {. }\end{array}$ \\
\hline $\mathrm{L} 2\left(l_{i}, l_{j}\right)$ & $\begin{array}{l}\text { Distance function between the layers } l_{i}, l_{j} \\
\mathrm{~L} 2\left(l_{i}, l_{j}\right)=\left|x_{i}-x_{j}\right|+\left|y_{i}-y_{j}\right|+\left|z_{i}-z_{j}\right| f_{c} \text {, where } f_{c}(\cdot) \\
\text { is a cost function between the layers. }\end{array}$ \\
\hline $\mathcal{D}_{l_{i}, l_{j}}^{A\left(C^{l_{i}, k}\right)}\left(C^{l_{i}, k}, C^{l_{j}, k}\right)$ & $\begin{array}{l}\text { Interlayer interference at full overlapping circuits } C^{l_{i}, k} \text { and } C^{l_{j}, k} \\
\text { between layers } l_{i}, l_{j} \text {. }\end{array}$ \\
\hline
\end{tabular}


Table 1 continued

\begin{tabular}{|c|c|}
\hline Notation & Description \\
\hline $\mathcal{D}_{l_{i}, l_{j}}^{\bar{A}\left(C^{l_{i}, k}, C^{l_{j}, k}\right)}\left(C^{l_{i}, k}, C^{l_{j}, k}\right)$ & $\begin{array}{l}\text { Interlayer interference at partial overlapping circuits } C^{l_{i}, k} \text { and } \\
C^{l_{j}, k} \text { between two neighboring layers } l_{i}, l_{j} \text {. }\end{array}$ \\
\hline $\mathcal{D}_{l_{i}, l_{j}}^{0}\left(C^{l_{i}, k}, C^{l_{j}, k}\right)$ & $\begin{array}{l}\text { Interlayer interference at zero overlapping circuits } C^{l_{i}, k} \text { and } C^{l_{j}, k} \\
\text { between two neighboring layers } l_{i}, l_{j}, \bar{A}\left(C^{l_{i}, k}, C^{l_{j}, k}\right)=0 \text {. }\end{array}$ \\
\hline$h\left(C^{l_{i}, k}, C^{l_{j}, k}\right)$ & Horizontal distance between $C^{l_{i}, k}$ and $C^{l_{j}, k}$. \\
\hline 5 & Constant (decay factor) in $\mathcal{D}_{l_{i}, l_{j}}^{0}\left(C^{l_{i}, k}, C^{l_{j}, k}\right)$. \\
\hline$h\left(C^{l_{i}, k}, C^{l_{i}, f}\right)$ & $\begin{array}{l}\text { Horizontal distance between two parallel running circuits } C^{l_{i}, k} \text { and } \\
C^{l_{i}, f} \text { in layer } l_{i} \text {. }\end{array}$ \\
\hline$\Phi\left(h\left(C^{l_{i}, k}, C^{l_{j}, k}\right)\right)$ & Coupling capacitance coefficient. \\
\hline$v\left(C^{l_{i}, k}, C^{l_{j}, k}\right)$ & $\begin{array}{l}\text { Vertical distance of two perpendicular running circuits } C^{l_{i}, k} \text { and } \\
C^{l_{i}, f} \text { in the same layer } l_{i} \text {. }\end{array}$ \\
\hline $\mathcal{D}_{l_{i}, l_{j}}^{0}\left(C^{l_{i}, k}, C^{l_{i}, f}\right)$ & Intralayer interference between $C^{l_{i}, k}$ and $C^{l_{i}, f}$ in layer $l_{i}$. \\
\hline$\chi_{k}, \lambda_{l_{i}}$ & Parameters of algorithm $\mathcal{A}_{3}$. \\
\hline$\alpha\left(U_{j}\right), \varphi\left(U_{j}\right), \Phi\left(U_{j}\right)$ & Interference coefficients of $U_{j}$. \\
\hline$\Omega\left(U_{j}\right)$ & $\begin{array}{l}\text { Sum of interference coefficients of } U_{j} \text {, as } \\
\quad \Omega\left(U_{j}\right)=\alpha\left(U_{j}\right)+\varphi\left(U_{j}\right)+\Phi\left(U_{j}\right) .\end{array}$ \\
\hline $\mathcal{D}\left(U_{j}\right)$ & $\begin{array}{l}\text { Gaussian noise of } U_{j} \text { (decoherence of the Josephson energy } E_{J} \text { of } \\
U_{j} \text { ). }\end{array}$ \\
\hline$H\left(\mathcal{C}_{q}\right)$ & Hamiltonian of $\mathcal{C}_{q}$. \\
\hline$E_{C}$ & Charging energy. \\
\hline$C_{J}$ & Capacitance of the Josephson junction. \\
\hline$n$ & Number of Cooper-pairs to tunnel the junction $J$. \\
\hline$n_{g}$ & Charge on the capacitor in units of Cooper pairs number. \\
\hline$\phi$ & Superconducting wave function phase difference across $J$. \\
\hline$|\psi\rangle$ & System state of $U_{j}$. \\
\hline$\hat{T}$ & Time-ordering operator. \\
\hline$\hbar$ & Reduced Planck constant. \\
\hline$f_{0}$ & Angular frequency. \\
\hline $\bar{E}_{J}$ & Mean of $E_{J}$ of $U_{j}$ \\
\hline$\sigma_{E_{J}}$ & $\begin{array}{l}\text { Standard deviation of } E_{J}, \sigma_{E_{J}}=\Delta E_{J}=\left|E_{J}^{(0)}-E_{J}^{(1)}\right| \text {, where } \\
\Delta E_{J} \text { is the width of the Gaussian distribution of } E_{J} \text { centered on } \\
\bar{E}_{J} \text {, and } E_{J}^{(0)} \text { and } E_{J}^{(1)} \text { are parameterize the width } \Delta E_{j} \text {. }\end{array}$ \\
\hline$C^{\prime}$ & Capacitance $C$ of $U_{j}$ at any nonzero $\Omega\left(U_{j}\right), C^{\prime}=C+\Omega\left(U_{j}\right)$. \\
\hline$E_{C}^{\prime}$ & Charging energy at any nonzero $\Omega\left(U_{j}\right)$. \\
\hline
\end{tabular}




\section{Table 1 continued}

\begin{tabular}{ll}
\hline Notation & Description \\
\hline$n_{g}^{\prime}$ & Charge on the capacitor in units of Cooper pairs number at any \\
& nonzero $\Omega\left(U_{j}\right)$. \\
$\sigma_{E_{j}^{\prime}}$ & Standard deviation of Josephson energy at any nonzero $\Omega\left(U_{j}\right)$. \\
$\bar{E}_{J}^{\prime}$ & Mean of Josephson energy at any nonzero $\Omega\left(U_{j}\right)$. \\
$\zeta$ & Time constant of exponentially decreasing probability function, \\
& $\zeta=\frac{\sigma_{E}}{\hbar}$, where $\sigma_{E_{J}}$ is the standard deviation of $\mathcal{D}\left(U_{j}\right)$ in the \\
& Josephson energy. \\
$\zeta^{\prime}$ & Time constant of exponentially decreasing probability function at \\
& any nonzero $\Omega\left(U_{j}\right), \zeta^{\prime}=\frac{\sigma_{E_{J}}^{\prime}}{\hbar}=\frac{\sigma_{j}+\sigma_{\Omega}}{\hbar}=\frac{\Delta E_{J}^{\prime}}{\hbar}$. \\
$f^{\prime}$ & Oscillation frequency at any nonzero $\Omega\left(U_{j}\right), f^{\prime}=\frac{\bar{E}_{J}^{\prime}}{\hbar}$. \\
$\mathcal{D}\left(U_{j}\right)_{\Omega\left(U_{j}\right)}$ & Gaussian noise of $U_{j}$ at a nonzero $\Omega\left(U_{j}\right)$. \\
$\tilde{\Omega}\left(U_{j}\right), \tilde{\mathcal{D}}\left(U_{j}\right)$ & Parameters of algorithm $\mathcal{A}_{4}$. \\
$\Delta$ & Abs. value of the difference of means $\bar{E}_{J}^{\prime}$ and $\bar{E}_{J}, \Delta=\left|\bar{E}_{J}^{\prime}-\bar{E}_{J}\right|$. \\
$\gamma$ & Abs. value of the difference of standard deviations $\Delta E_{J}^{\prime}$ and $\Delta E_{J}$, \\
& $\gamma=\left|\Delta E_{J}^{\prime}-\Delta E_{J}\right|$.
\end{tabular}




\section{References}

1. Arute, F., et al.: Quantum supremacy using a programmable superconducting processor. Nature 574, 505-510 (2019). https://doi.org/10.1038/s41586-019-1666-5

2. Preskill, J.: Quantum computing in the NISQ era and beyond. Quantum 2, 79 (2018)

3. Harrow, A.W., Montanaro, A.: Quantum computational supremacy. Nature 549, 203-209 (2017)

4. Aaronson, S., Chen, L.: Complexity-theoretic foundations of quantum supremacy experiments. In: Proceedings of the 32nd Computational Complexity Conference, CCC '17, pp. 22:1-22:67 (2017)

5. Alexeev, Y. et al.: Quantum computer systems for scientific discovery, arXiv:1912.07577 (2019)

6. Loncar, M. et al.: Development of quantum interConnects for next-generation information technologies, arXiv:1912.06642 (2019)

7. Ajagekar, A., Humble, T., You, F.: Quantum computing based hybrid solution strategies for large-scale discrete-continuous optimization problems. Comput. Chem. Eng. 132, 106630 (2020)

8. Ajagekar, A., You, F.: Quantum computing for energy systems optimization: challenges and opportunities. Energy 179, 76-89 (2019)

9. IBM: A new way of thinking: The IBM quantum experience. http://www.research.ibm.com/quantum (2017)

10. Harrigan, M. et al.: Quantum approximate optimization of non-planar graph problems on a planar superconducting processor, arXiv:2004.04197v1 (2020)

11. Rubin, N. et al.: Hartree-Fock on a superconducting qubit quantum computer, arXiv:2004.04174v1 (2020)

12. Lloyd, S.: Quantum approximate optimization is computationally universal, arXiv:1812.11075 (2018)

13. Sax, I. et al.: Approximate approximation on a quantum annealer, arXiv:2004.09267 (2020)

14. Brown, K.A., Roser, T.: Towards storage rings as quantum computers. Phys. Rev. Accel. Beams 23, 054701 (2020)

15. Gyongyosi, L., Imre, S., Nguyen, H.V.: A Survey on Quantum Channel Capacities. IEEE Commun. Surv. Tutor. 99, 1 (2018). https://doi.org/10.1109/COMST.2017.2786748

16. Gyongyosi, L., Imre, S.: A survey on quantum computing technology, Computer Science Review, Elsevier, https://doi.org/10.1016/j.cosrev.2018.11.002, ISSN: 1574-0137 (2018)

17. Debnath, S., et al.: Demonstration of a small programmable quantum computer with atomic qubits. Nature 536, 63-66 (2016)

18. Barends, R., et al.: Superconducting quantum circuits at the surface code threshold for fault tolerance. Nature 508, 500-503 (2014)

19. Monz, T., et al.: Realization of a scalable Shor algorithm. Science 351, 1068-1070 (2016)

20. DiCarlo, L., et al.: Demonstration of two-qubit algorithms with a superconducting quantum processor. Nature 460, 240-244 (2009)

21. Higgins, B.L., Berry, D.W., Bartlett, S.D., Wiseman, H.M., Pryde, G.J.: Entanglement-free Heisenberg-limited phase estimation. Nature 450, 393-396 (2007)

22. Kielpinski, D., Monroe, C., Wineland, D.J.: Architecture for a large-scale ion-trap quantum computer. Nature 417, 709-711 (2002)

23. Ofek, N., et al.: Extending the lifetime of a quantum bit with error correction in superconducting circuits. Nature 536, 441-445 (2016)

24. Gulde, S., et al.: Implementation of the Deutsch-Jozsa algorithm on an ion-trap quantum computer. Nature 421, 48-50 (2003)

25. Farhi, E., Neven, H.: Classification with quantum neural networks on near term processors, arXiv:1802.06002v1 (2018)

26. Yuan, Z., Chen, Y., Zhao, B., Chen, S., Schmiedmayer, J., Pan, J.-W.: Nature 454, 1098-1101 (2008)

27. Biamonte, J., et al.: Quantum machine learning. Nature 549, 195-202 (2017)

28. Lloyd, S., Mohseni, M., Rebentrost, P.: Quantum principal component analysis. Nat. Phys. 10, 631 (2014)

29. Sheng, Y.B., Zhou, L.: Distributed secure quantum machine learning. Science 62, 1025-2019 (2017)

30. Kimble, H.J.: The quantum Internet. Nature 453, 1023-1030 (2008)

31. Lloyd, S., Shapiro, J.H., Wong, F.N.C., Kumar, P., Shahriar, S.M., Yuen, H.P.: Infrastructure for the quantum internet. ACM SIGCOMM Comput. Commun. Rev. 34, 9-20 (2004)

32. Van Meter, R.: Quantum Networking. Wiley, New York (2014)

33. Imre, S., Gyongyosi, L.: Advanced Quantum Communications - An Engineering Approach. WileyIEEE Press, New Jersey, USA (2013) 
34. Pirandola, S.: Capacities of repeater-assisted quantum communications, arXiv:1601.00966 (2016)

35. Bacsardi, L.: On the way to quantum-based satellite communication. IEEE Commun. Mag. 51(08), 50-55 (2013)

36. Petz, D.: Quantum Information Theory and Quantum Statistics. Springer-Verlag, Heidelberg (2008)

37. Farhi, E., Goldstone, J., Gutmann, S.: A quantum approximate optimization algorithm. arXiv:1411.4028 (2014)

38. Farhi, E., Goldstone, J., Gutmann, S., Neven, H.: Quantum algorithms for fixed qubit architectures. arXiv:1703.06199v1 (2017)

39. Farhi, E., Goldstone, J., Gutmann, S.: A quantum approximate optimization algorithm applied to a bounded occurrence constraint problem. arXiv:1412.6062 (2014)

40. Farhi, E., Harrow, A.H.: Quantum supremacy through the quantum approximate optimization algorithm. arxiv:1602.07674 (2016)

41. Gyongyosi, L.: Quantum state optimization and computational pathway evaluation for gate-model quantum computers. Scientific Reports (2020). https://doi.org/10.1038/s41598-020-61316-4

42. Gyongyosi, L.: Unsupervised quantum gate control for gate-model quantum computers. Scientific Reports (2020). https://doi.org/10.1038/s41598-020-67018-1

43. Gyongyosi, L., Imre, S.: Circuit depth reduction for gate-model quantum computers. Scientific Reports (2020). https://doi.org/10.1038/s41598-020-67014-5

44. Gyongyosi, L.: Objective function estimation for solving optimization problems in gate-model quantum computers. Scientific Reports (2020). https://doi.org/10.1038/s41598-020-71007-9

45. Gyongyosi, L., Imre, S.: Quantum circuit design for objective function maximization in gate-model quantum computers. Quantum Inf. Process. (2019) https://doi.org/10.1007/s11128-019-2326-2

46. Gyongyosi, L., Imre, S.: State stabilization for gate-model quantum computers. Quantum Inf. Process. (2019) https://doi.org/10.1007/s11128-019-2397-0

47. Gyongyosi, L., Imre, S.: Dense quantum measurement theory, Scientific Reports. Nature (2019). https://doi.org/10.1038/s41598-019-43250-2

48. Gyongyosi, L., Imre, S.: Training optimization for gate-model quantum neural networks, Scientific Reports. Nature (2019). https://doi.org/10.1038/s41598-019-48892-w

49. Gyongyosi, L., Imre, S.: Optimizing high-efficiency quantum memory with quantum machine learning for near-term quantum devices, Scientific Reports. Nature (2020). https://doi.org/10.1038/s41598019-56689-0

50. Zhou, X., Leung, D.W., Chuang, I.L.: Methodology for quantum logic gate construction. Phys. Rev. A 62, 052316 (2000)

51. Amy, M., Maslov, D., Mosca, M., Roetteler, M.: A meet-in-the middle algorithm for fast synthesis of depth-optimal quantum circuits. IEEE Trans. Comput.-Aided Design Integr. Circuits Syst. 32(6), 818-830 (2013)

52. Paler, A., Polian, I., Nemoto, K., Devitt, S.J.: Fault-tolerant, high level quantum circuits: form, compilation and description. Quantum Sci. Tech. 2(2), 025003 (2017)

53. Brandao, F.G.S.L., Broughton, M., Farhi, E., Gutmann, S., Neven, H.: For fixed control parameters the quantum approximate optimization algorithm's objective function value concentrates for typical instances, arXiv:1812.04170 (2018)

54. Zhou, L., Wang, S.-T., Choi, S., Pichler, H., Lukin, M.D.: Quantum approximate optimization algorithm: performance, mechanism, and implementation on near-term devices, arXiv:1812.01041 (2018)

55. Lechner, W.: Quantum approximate optimization with parallelizable gates, arXiv:1802.01157v2 (2018)

56. Crooks, G.E.: Performance of the quantum approximate optimization algorithm on the maximum cut problem, arXiv:1811.08419 (2018)

57. Ho, W. W., Jonay, C. and Hsieh, T.H.: Ultrafast state preparation via the quantum approximate optimization algorithm with long range interactions, arXiv:1810.04817 (2018)

58. Song, C., et al.: 10-Qubit entanglement and parallel logic operations with a superconducting circuit. Phys. Rev. Lett. 119(18), 180511 (2017)

59. Farhi, E., Kimmel, S., Temme, K.: A Quantum Version of Schoning's Algorithm Applied to Quantum 2-SAT, arXiv:1603.06985 (2016)

60. Farhi, E., Gamarnik, D., Gutmann, S.: The quantum approximate optimization algorithm needs to see the whole graph: a typical case, arXiv:2004.09002v1 (2020)

61. Farhi, E., Gamarnik, D., Gutmann, S.: The quantum approximate optimization algorithm needs to see the whole graph: worst case examples, arXiv:2005.08747 (2020) 
62. Farhi, E., Goldstone, J., Gutmann, S., Zhou, L.: The quantum approximate optimization algorithm and the Sherrington-Kirkpatrick model at infinite size, arXiv:1910.08187 (2019)

63. Kjaergaard, M., et al.: Superconducting Qubits: Current State of Play. Ann. Rev. Condens. Matter Phys. 11, 369-395 (2020)

64. Pirandola, S., Laurenza, R., Ottaviani, C., Banchi, L.: Fundamental limits of repeaterless quantum communications. Nat. Commun. 15043, (2017). https://doi.org/10.1038/ncomms 15043

65. Pirandola, S., Braunstein, S.L., Laurenza, R., Ottaviani, C., Cope, T.P.W., Spedalieri, G., Banchi, L.: Theory of channel simulation and bounds for private communication. Quantum Sci. Technol. 3, 035009 (2018)

66. Pirandola, S.: End-to-end capacities of a quantum communication network. Commun. Phys. 2, 51 (2019)

67. Pirandola, S., Braunstein, S.L.: Unite to build a quantum internet. Nature 532, 169-171 (2016)

68. Pirandola, S.: Bounds for multi-end communication over quantum networks. Quantum Sci. Technol. 4, 045006 (2019)

69. Pirandola, S. et al.: Advances in Quantum Cryptography, arXiv:1906.01645 (2019)

70. Laurenza, R., Pirandola, S.: General bounds for sender-receiver capacities in multipoint quantum communications. Phys. Rev. A 96, 032318 (2017)

71. Caleffi, M.: End-to-end entanglement rate: toward a quantum route metric, 2017 IEEE Globecom (2018) https://doi.org/10.1109/GLOCOMW.2017.8269080

72. Caleffi, M.: Optimal routing for quantum networks. IEEE Access 5, (2017). https://doi.org/10.1109/ ACCESS.2017.2763325

73. Caleffi, M., Cacciapuoti, A. S., Bianchi, G.: Quantum internet: from communication to distributed computing, arXiv:1805.04360 (2018)

74. Castelvecchi, D.: The quantum internet has arrived, Nature, News and Comment (2018) https://www. nature.com/articles/d41586-018-01835-3

75. Cacciapuoti, A.S., Caleffi, M., Tafuri, F., Cataliotti, F.S., Gherardini, S., Bianchi, G.: Quantum internet: networking challenges in distributed quantum computing arXiv:1810.08421 (2018)

76. Wehner, S., Elkouss, D., Hanson, R.: Quantum internet: A vision for the road ahead. Science 362, 6412 (2018)

77. Cuomo, D., Caleffi, M., Cacciapuoti, A.S.: Towards a distributed quantum computing ecosystem, arXiv:2002.11808v1 (2020)

78. Quantum Internet Research Group (QIRG), web: https://datatracker.ietf.org/rg/qirg/about/ (2018)

79. Khatri, S.: Policies for elementary link generation in quantum networks, arXiv:2007.03193 (2020)

80. Miguel-Ramiro, J., Pirker, A., Dur, W.: Genuine quantum networks: superposed tasks and addressing, arXiv:2005.00020v1 (2020)

81. Pirker, A., Dur, W.: A quantum network stack and protocols for reliable entanglement-based networks, arXiv:1810.03556v1 (2018)

82. Shannon, K., Towe, E., Tonguz, O.: On the use of quantum entanglement in secure communications: a survey, arXiv:2003.07907 (2020)

83. Amoretti, M., Carretta, S.: Entanglement verification in quantum networks with tampered nodes. IEEE J. Sel. Areas Commun. (2020). https://doi.org/10.1109/JSAC.2020.2967955

84. Cao, Y., et al.: Multi-tenant provisioning for quantum key distribution networks with heuristics and reinforcement learning: a comparative study. IEEE Trans. Netw. Serv. Manag. (2020). https://doi.org/ 10.1109/TNSM.2020.2964003

85. Cao, Y., et al.: Key as a service (KaaS) over quantum key distribution (QKD)-integrated optical networks. IEEE Commun. Mag. (2019). https://doi.org/10.1109/MCOM.2019.1701375

86. Liu, Y.: Preliminary study of connectivity for quantum key distribution network, arXiv:2004.11374v1 (2020)

87. Amer, O., Krawec, W.O., Wang, B.: Efficient routing for quantum key distribution networks, arXiv:2005.12404 (2020)

88. Sun, F.: Performance analysis of quantum channels, Quantum Eng. e35, https://doi.org/10.1002/que2. 35(2020)

89. Chai, G. et al.: Blind channel estimation for continuous-variable quantum key distribution, Quantum Eng., e37, https://doi.org/10.1002/que2.37(2020)

90. Ahmadzadegan, A.: Learning to utilize correlated auxiliary classical or quantum noise, arXiv:2006.04863v1 (2020)

91. Bausch, J.: Recurrent quantum neural networks, arXiv:2006.14619v1 (2020) 
92. Xin, T.: Improved quantum state tomography for superconducting quantum computing systems, arXiv:2006.15872v1 (2020)

93. Dong, K., et al.: Distributed subkey-relay-tree-based secure multicast scheme in quantum data center networks. Optical Eng. 59(6), 065102 (2020). https://doi.org/10.1117/1.OE.59.6.065102

94. Gyongyosi, L.: Services for the quantum internet, DSc Dissertation, Hungarian Academy of Sciences (MTA) (2020)

95. Krisnanda, T., et al.: Probing quantum features of photosynthetic organisms. NPJ Quantum Inf. 4, 60 (2018)

96. Krisnanda, T., et al.: Revealing nonclassicality of inaccessible objects. Phys. Rev. Lett. 119, 120402 (2017)

97. Krisnanda, T., et al.: Observable quantum entanglement due to gravity. NPJ Quantum Inf. 6, 12 (2020)

98. Krisnanda, T., et al.: Detecting nondecomposability of time evolution via extreme gain of correlations. Phys. Rev. A 98, 052321 (2018)

99. Krisnanda, T.: Distribution of quantum entanglement: principles and applications, PhD Dissertation, Nanyang Technological University, arXiv:2003.08657 (2020)

100. Ghosh, S. et al.: Universal quantum reservoir computing. arXiv:2003.09569 (2020)

101. Komarova, K., et al.: Quantum device emulates dynamics of two coupled oscillators. J. Phys. Chem. Lett. (2020). https://doi.org/10.1021/acs.jpclett.0c01880

102. Gattuso, H., et al.: Massively parallel classical logic via coherent dynamics of an ensemble of quantum systems with dispersion in size. ChemRxiv. Preprint (2020). https://doi.org/10.26434/chemrxiv. 12370538.v1

103. Chessa, S., Giovannetti, V.: Multi-level amplitude damping channels: quantum capacity analysis, arXiv:2008.00477 (2020)

104. Pozzi, M.G. et al.: Using reinforcement learning to perform qubit routing in quantum compilers, arXiv:2007.15957 (2020)

105. Bartkiewicz, K., et al.: Experimental kernel-based quantum machine learning in finite feature space. Sci. Rep. 10, 12356 (2020). https://doi.org/10.1038/s41598-020-68911-5

106. Chakraborty, K., Rozpedeky, F., Dahlbergz, A., Wehner, S.: Distributed routing in a quantum internet, arXiv:1907.11630v1 (2019)

107. Khatri, S., Matyas, C.T., Siddiqui, A.U., Dowling, J.P.: Practical figures of merit and thresholds for entanglement distribution in quantum networks. Phys. Rev. Res. 1, 023032 (2019)

108. Kozlowski, W., Wehner, S.: Towards large-scale quantum networks, In: Proceeding of the Sixth Annual ACM International Conference on Nanoscale Computing and Communication, Dublin, Ireland, arXiv:1909.08396 (2019)

109. Pathumsoot, P., Matsuo, T., Satoh, T., Hajdusek, M., Suwanna, S., Van Meter, R.: Modeling of measurement-based quantum network coding on IBMQ devices. Phys. Rev. A 101, 052301 (2020)

110. Pal, S., Batra, P., Paterek, T., Mahesh, T.S.: Experimental localisation of quantum entanglement through monitored classical mediator, arXiv:1909.11030v1 (2019)

111. Miguel-Ramiro, J., Dur, W.: Delocalized information in quantum networks. New J. Phys (2020). https://doi.org/10.1088/1367-2630/ab784d

112. Gyongyosi, L.: Dynamics of entangled networks of the quantum internet. Sci. Rep (2020). https:// doi.org/10.1038/s41598-020-68498-x

113. Gyongyosi, L. Imre, S.: Routing space exploration for scalable routing in the quantum internet. Sci. Rep (2020). https://doi.org/10.1038/s41598-020-68354-y

114. Mewes, L., Wang, M., Ingle, R.A., et al.: Energy relaxation pathways between light-matter states revealed by coherent two-dimensional spectroscopy. Commun. Phys 3, 157 (2020)

115. Guo, D., et al.: Comprehensive high-speed reconciliation for continuous-variable quantum key distribution. Quantum Inf. Process 19, 320 (2020)

116. Chen, L., Hu, M.: Locally maximally mixed states. Quantum Inf. Process 19, 305 (2020)

117. Kopszak, P., Mozrzymas, M., Studzinski, M.: Positive maps from irreducibly covariant operators. J. Phys. A: Math. Theor. 53, 395306 (2020)

118. Barbeau, M. et al.: Capacity requirements in networks of quantum repeaters and terminals. In: Proceedings of IEEE International Conference on Quantum Computing and Engineering (QCE 2020) (2020)

119. Yin, J., et al.: Entanglement-based secure quantum cryptography over 1,120 kilometres. Nature 582, 501 (2020) 
120. Santra, S. and Malinovsky, V. S. Quantum networking with shortrange entanglement assistance. arXiv:2008.05553 (2020)

121. Rodrigues, D.A.: Superconducting Charge Qubits, Ph.D. Dissertation, H. H. Wills Physics Laboratory, University of Bristol (2003)

122. Shnirman, A., Schon, G., Herman, Z.: Quantum manipulations of small josephson junctions. Phys. Rev. Lett. 79, 2371 (1997)

123. Makhlin, Y., Schon, G., Shnirman, A.: Josephson-junction qubits with controlled couplings. Nature 398, 305 (1999)

124. Makhlin, Y., Schon, G., Shnirman, A.: Nano-electronic realizations of quantum bits. J. Low Temp. Phys. 118, $751(2000)$

125. Oh, S.: Errors due to finite rise and fall times of pulses in superconducting charge qubits. Phys. Rev. B 65, 144526 (2002)

126. Paladino, E., Faoro, L., Falci, G., Fazio, R.: Decoherence and 1/f Noise in Josephson Qubits. Phys. Rev. Lett. 188, 228304 (2002)

127. van der Wal, C.H., Wilhelm, F.K., Harmans, C.J.P.M., Mooij, J.E.: Engineering decoherence in Josephson persistent-current qubits: Measurement apparatus and other electromagnetic environments. Eur. Phys. J. B 31, 111 (2003)

128. Scovell, R.W., et al.: Quantum states of small superconductors. IEEE Proc.-Sci. Meas. Technol. 148, 233-236 (2001)

129. Martins, R., Lourenco, N., Horta, N.: Analog Integrated Circuit Design Automation, Springer, ISBN 978-3-319-34059-3, ISBN 978-3-319-34060-9 (2017)

130. Martins, R., Lourenco, N., Horta, N.: Multi-objective optimization of analog integrated circuit placement hierarchy in absolute coordinates. Expert Syst. Appl. 42(23), 9137-9151 (2015)

131. Martins, R., Povoa, R., Lourenco, N., Horta, N.: Current-flow and current-density-aware multiobjective optimization of analog IC placement. Integr, VLSI J (2016)

132. Chang, Y.-C., Chang, Y.-W., Wu, G.-M., Wu, S.-W.: B*-trees: A new representation for nonslicing floorplans, In: Proceedings of the 37th ACM/IEEE Design Automation Conference (DAC), pp. 458463 (2000)

133. Chang, Y.-W.: A binary-tree modeling of non-slicing floorplans, Online version: http://www.cc.ee. ntu.edu.tw/ ywchang/Papers (2004)

134. Bandyopadhyay, S., Saha, S., Maulik, U., Deb, K.: A simulated annealing-based multiobjective optimization algorithm: AMOSA. IEEE Trans. Evol. Comput. 12(3), 269-283 (2008)

135. Suman, B., Kumar, P.: A survey of simulated annealing as a tool for single and multiobjective optimization. J. Oper. Res. Soc. 57, 1143-1160 (2006)

136. Foxen, B. et al.: Demonstrating a continuous set of two-qubit gates for near-term quantum algorithms, arXiv:2001.08343 (2020)

Publisher's Note Springer Nature remains neutral with regard to jurisdictional claims in published maps and institutional affiliations. 